Published in final edited form as:

Nat Mater. 2017 June ; 16(6): 671-680. doi:10.1038/nmat4866.

\title{
Colony Stimulating Factor-1 Receptor is a central component of the foreign body response to biomaterial implants in rodents and non-human primates
}

\author{
Joshua C. Doloff ${ }^{1,2,3}$, Omid Veiseh ${ }^{1,2,3,4}$, Arturo J. Vegas ${ }^{1,2,5}$, Hok Hei Tam ${ }^{1,3}$, Shady \\ Farah $^{1,2,3}$, Minglin Ma1,2,6, Jie Li ${ }^{1,2}$, Andrew Bader ${ }^{1,2}$, Alan Chiu ${ }^{1,2}$, Atieh Sadraei ${ }^{1}$, \\ Stephanie Aresta-Dasilva ${ }^{1,2}$, Marissa Griffin ${ }^{1}$, Siddharth Jhunjhunwala ${ }^{1,2}$, Matthew \\ Webber $^{1,3}$, Sean Siebert ${ }^{1,2}$, Katherine Tang ${ }^{1,2}$, Michael Chen ${ }^{1,2}$, Erin Langan ${ }^{1,2}$, Nimit \\ Dholokia $^{1,2}$, Raj Thakrarr, ${ }^{1,2}$, Meirigeng $\mathbf{Q i}^{7}$, Jose Oberholzer ${ }^{7}$, Dale L. Greiner ${ }^{8}$, Robert \\ Langer ${ }^{1,2,3,9,10,11}$, and Daniel G. Anderson ${ }^{1,2,3,9,10,11,{ }^{*}}$ \\ ${ }^{1}$ David H. Koch Institute for Integrative Cancer Research, Massachusetts Institute of Technology, \\ 500 Main Street, Cambridge, MA, 02139, USA \\ 2Department of Anesthesiology, Boston Children's Hospital, 300 Longwood Ave, Boston, MA \\ 02115, USA \\ ${ }^{3}$ Department of Chemical Engineering, Massachusetts Institute of Technology, 77 Massachusetts \\ Avenue, Cambridge, MA, 02139, USA \\ ${ }^{7}$ Division of Transplantation, Department of Surgery, University of Illinois at Chicago, Chicago, IL \\ ${ }^{8}$ Program in Molecular Medicine, University of Massachusetts Medical School, Worcester, MA \\ 01605, USA \\ ${ }^{9}$ Division of Health Science Technology, Massachusetts Institute of Technology, 77 Massachusetts \\ Avenue, Cambridge, MA, 02139, USA \\ ${ }^{10}$ Institute for Medical Engineering and Science, Massachusetts Institute of Technology, 77 \\ Massachusetts Avenue, Cambridge, MA, 02139, USA
}

Users may view, print, copy, and download text and data-mine the content in such documents, for the purposes of academic research, subject always to the full Conditions of use: http://www.nature.com/authors/editorial_policies/license.html\#terms

*dgander@mit.edu; Tel.: +1 6172586843 ; fax: +1 6172588827.

${ }_{5}^{4}$ Current address: Sigilon, Inc., 161 First St., Cambridge, MA 02142, USA

${ }^{5}$ Current address: Department of Chemistry, Boston University, Boston, Massachusetts, USA

${ }^{6}$ Current address: Biological and Environmental Engineering, Cornell University, Ithaca, NY 14853, USA

DATA AVAILABILITY

All data generated during this study are included in this published article (and its Supplementary Information).

SUPPLEMENTARY INFORMATION

Supplementary Tables $1-3$

Supplementary Figure S1-S20

COMPETING FINANCIAL INTERESTS

The authors declare no competing financial interests.

AUTHOR CONTRIBUTIONS

J.D. and D.A. designed experiments, analyzed data, and wrote the manuscript. J.D., O.V., A.V., H.H.T., S.F., M.M., J.L., A.B., A.C., S.A-D., M.G., A.S., S.J., M.W., S.S., K.T., M.C., E.L., N.D., R.T., M.Q. and J.O. performed experiments. J.D. and H.T. performed statistical analyses of data sets and aided in the preparation of displays communicating data sets. J.O. and D.G. provided conceptual advice and technical support. R.L. and D.A. supervised the study. All authors discussed the results and assisted in the preparation of the manuscript. 
${ }^{11}$ Harvard-MIT Division of Health Science and Technology, Massachusetts Institute of Technology, 77 Massachusetts Avenue, Cambridge, MA, 02139, USA

\section{Abstract}

Host recognition and immune-mediated foreign body response (FBR) to biomaterials can compromise the performance of implanted medical devices. To identify key cell and cytokine targets, here we perform in-depth systems analysis of innate and adaptive immune system responses to implanted biomaterials in rodents and non-human primates. While macrophages are indispensable to the fibrotic cascade, surprisingly neutrophils and complement are not. Macrophages, via CXCL13, lead to downstream B cell recruitment, which further potentiated fibrosis, as confirmed by B cell knock out and CXCL13 neutralization. Interestingly, Colony Stimulating Factor-1 Receptor (CSF1R) is significantly increased following implantation of multiple biomaterial classes: ceramic, polymer, and hydrogel. Its inhibition, like macrophage depletion, leads to complete loss of fibrosis, but spares other macrophage functions such as wound healing, ROS production, and phagocytosis. Our results indicate targeting CSF1R may allow for a more selective method of fibrosis inhibition, and improve biomaterial biocompatibility without the need for broad immunosuppression.

Implanted biomedical devices reside within tens of millions of patients in the United States alone, and are involved in millions of new and revisionary surgeries every year ${ }^{1,2}$. As such, they comprise a major component of modern medicine and are essential for many clinical applications ranging from hip/knee replacement ${ }^{3}$, tissue repair/reconstruction ${ }^{4}$, prosthesis and neural interfacing ${ }^{5}$, controlled drug release ${ }^{6}$, electronic pacing ${ }^{7}$, vital sign monitoring ${ }^{8}$, intraocular lens replacement ${ }^{9}$, and cell encapsulation and transplantation ${ }^{10}$. Unlike particulates, which may be phagocytosed and cleared by the innate immune system ${ }^{3}$, larger non-biodegradable macroscale devices remain in the body. The host senses these implants as foreign and mounts an immune-mediated rejection response ${ }^{11,12}$. This sequence, known as the fibrotic cascade, includes tissue injury, immune recruitment and adhesion, myofibroblast induction, and fibrous capsule formation ${ }^{12}$, preventing interaction with the surrounding microenvironment, including sensing of biochemical stimuli such as $\mathrm{pH}$, oxygen, blood glucose levels, and/or obstructing nutrient flux where internal device components are of biologic origin ${ }^{11,13}$.

Aspects of the biology involved in the ensuing immune response have been characterized $^{11,13}$. Macrophages have remarkable plasticity, responding to numerous signals ${ }^{14}$, and are a key component of material recognition, actively adhering to the surface of foreign objects $11,13,15,16$. They are increased locally throughout the implant site within days and may persist at the material surface ${ }^{11,13,16}$. Device contact initiates macrophage fusion into foreign-body giant cells ${ }^{11,17}$, which recruit fibroblasts responsible for final fibrous collagen and matrix protein deposition ${ }^{11,13,18}$. This fate is the same for materials of both natural and synthetic origin, including polysaccharides, polymers, ceramics, and metals $^{13}$.

One natural polysaccharide, alginate, is a multipurpose biomaterial that has been utilized in numerous biomedical applications including biosensors, tissue regeneration, cell 
encapsulation, and drug delivery ${ }^{10,19}$. While non-biodegradable alginate capsules are used to immunoisolate transplanted islets for type 1 diabetes therapy, host immune and fibrosis responses directed to the encapsulating biomaterial results in device failure ${ }^{20,21,22,23}$. Even empty alginate microspheres elicit rejection in some animal models ${ }^{24,25}$, including nonhuman primates ${ }^{26,27}$. This response has been a barrier to translation of alginateencapsulated islets for several decades 22,23 .

Overcoming the rejection of implanted biomaterial devices may allow for a range of medical advancements ${ }^{28,29}$. Current approaches for immune suppression or management of longterm biomedical device implantation often involve broad-spectrum anti-inflammatories ${ }^{30}$. The major immunosuppressive agents used as standard care for implantation procedures are rapamycin (sirolimus), tacrolimus, everolimus, cyclosporine, and corticosteroids ${ }^{31}$. However, many of these anti-inflammatory drugs are not specific to individual immune populations, having multiple targets and differential effects in vivo ${ }^{30,32}$. As such, more specific drug targets and corresponding inhibitory compounds need to be identified.

To do so, here we examine the roles of innate and adaptive immunity on biomaterial biocompatibility in vivo. While multiple materials are included for generalizability, we focus on alginate given its long history of use in the field of cell encapsulation, and the fact that the immune response to alginate remains a barrier to its success in the clinic ${ }^{20,21,22,23}$. Implantation of alginate into the intraperitoneal (IP) space of immune compliant BALB/c mice yields little to no fibrosis ${ }^{33,34}$, whereas alginate retrieved from C57BL/6 mice, which have more aggressive innate immunity, is densely covered with fibrous overgrowth ${ }^{33}$, mimicking the FBR observed in humans ${ }^{21,22,23}$ and non-human primates (NHPs) ${ }^{26,27}$. To dissect the immune pathways involved we examine a range of C57BL/6 rodents with different immune knockout or targeted perturbation/depletion states (see online Methods for full description). Immune cell and cytokine responses in these models are screened to identify more specific targets for inhibiting fibrosis. Several hits were additionally confirmed as relevant in cynomolgus monkeys.

\section{Alginate microspheres elicit a multi-immune population FBR}

Historically, FBRs to alginate were believed to be a function of contaminating endotoxins ${ }^{35}$. However, a number of studies indicate that clinical grade, commercially purified alginate does not contain detectable endotoxins ${ }^{26,27,36}$ (Supplementary Table S1). This indicates that the immune-mediated FBR is to biomaterial alginate itself, and is complex, engaging both innate and adaptive immune cell populations (Figure 1). Cellular adhesion and fibrotic overgrowth of $500 \mu \mathrm{m}$ alginate microspheres implanted in the intraperitoneal space of C57BL/6 mice after 14 days is apparent as a white plaque by phase contrast imaging (Figure 1a), with individual cells resolvable by nuclear DAPI staining (Figure 1b). A 14-day timepoint was selected in this study, as it has been established as suitable to resolve both immune and fibrotic responses to implanted materials, in both C57BL/6 mice and nonhuman primates ${ }^{26,27}$. Specific markers for innate immune cell macrophages (F4/80 and CD68) and neutrophils (Ly6g), adaptive immune B cells (CD19), as well as fibrosis of material implants (alpha smooth muscle actin (aSMactin) and collagen 1a1 (Col1a1)) were identified by qPCR, and increased over time on alginate spheres, as compared to non- 
implanted and mock (saline-injected) controls (Figure 1c and Supplemental Figure S1). Increases in aSMactin and Col1a1, as well as Col1a2, have previously been shown to increase proportionately to fibrotic encapsulation ${ }^{26,27}$, and as such are used here as markers. Despite the absence of implanted material for non-implant and mock surgery animals, omental and epididymal fat pads were taken for expression analysis, as these are the tissues to which materials are subsequently stuck and embedded within fibrotic tissue ${ }^{27}$. Immunofluorescent staining and confocal imaging of spheres retrieved 14 days postintraperitoneal (IP) implantation reveals that all three responding immune populations (macrophages: CD68; neutrophils, Ly6g/Gr1; B cells, CD19) reside within the fibrotic plaque directly enveloping implanted alginate spheres (Figure $1 \mathrm{~d} \&$ e). Intravital imaging at 14 days post-implant in Ccr6-EGFP transgenic C57BL/6 mice also verified trafficking of adaptive B cells onto implanted quantum-dot labeled (pink) alginate spheres (Figure 1f \& g).

\section{Characterization of local immune responses to alginate}

To determine whether the host immune response is acute and short-lived or manifested over a longer period of time, we performed FACS on cells retrieved at days 1, 4, 7, 14, and 28 following IP implantation into C57BL/6 mice (Figure 2a-c). In general, free-floating peritoneal cell numbers reflected an increase in neutrophils (as \% total), decrease in B cells, with macrophage levels unchanged over time (Figure 2a). However, these responses, representative of the liquid environment surrounding IP implants, do not accurately depict cell population increases identified directly on the implanted material surface (Figure 1 and Supplemental Figure S1).

Luminex analysis of serum cytokine levels following implantation showed an acute but transient increase of 4 out of 32 cytokines (Supplemental Figure S2) that went away within $\sim 1-3$ days post-implantation. This temporary response is in contrast to the longer-term evidence of immunogenicity of implanted alginate (Figure 2). Longer-term responses, unfortunately, do not show measurable global cytokine levels in circulating blood, as signals originating in inflamed tissues have likely become too dilute upon dissemination. As a more direct test, FACS was performed on dissociated, fibrosed IP tissue-immediately adjacent and stuck to fibrosed alginate (Supplemental Figure S3), as well as cells taken specifically from just the surface of implanted alginate spheres. FACS revealed that immune cells increased persistently over a 28-day period, with macrophages the major responding cell population (Figure $2 \mathrm{~b} \& \mathrm{c}$ ). Analysis of cells dissociated directly from the material surface at day 1 showed the presence of immature $\mathrm{CD} 68^{-} \mathrm{Gr} 1^{1 \mathrm{lo} /}-\mathrm{CD} 11 \mathrm{~b}^{+}$monocytes, known for being early responders patrolling out from the blood ${ }^{37}$. On days 4 and 7 , this immature population disappeared as mature $\mathrm{CD} 68^{+} \mathrm{CD} 11 \mathrm{~b}^{+}$macrophages increased in number (Figure 2c, purple vs red). Macrophage subtyping carried out on surface-dissociated cells at 1, 4, and 7 days also suggests this change is due to early-stage recruitment and differentiation, as macrophage phenotypes not only increased in magnitude but also changed in makeup over the first days following implantation ${ }^{27}$. Later stage (day 7 and beyond) recruitment of both adaptive immune $\mathrm{CD} 19^{+} \mathrm{IgM}^{+} \mathrm{B}$ cells and fibrosis-associated alpha smooth muscle actin (aSMactin)-positive myofibroblasts was observed (Figure 2c, white). Multiplexed NanoString gene expression analysis of lysates taken directly from alginate implants was 
also able to show longer-term increases (up to 4 weeks) in macrophage and neutrophil associated physical and cytokine markers (Figure 2d \& e).

Tissue immediately adjacent to fibrosed alginate (Supplemental Figure S3, epididymal and omental fat pads), as well as directly adhered to the material surface of free-floating, fibrosed spheres was analyzed over 28-days. Both showed increasing presence of macrophage marker CD68 and local inflammation marker TGF 31 (Supplemental Figure S4a $\&$ b), but a delayed increase in the B cell marker CD19 (Figure 2f). Lastly, increased expression of aSMactin, a marker for activated myofibroblasts responsible for downstream collagen deposition and fibrosis of material implants, was not observable in adjacent fibrosed tissue, due to background in IP fat pads (also the case in subcutaneous tissue). However, a significant increase, also starting at and increasing beyond day 7, was observed when fibrotic material was instead taken directly from the surface of implanted spheres (Figure $2 \mathrm{~g}$ ). These concomitant, delayed responses suggest a relationship between B cell and myofibroblast recruitment in the fibrotic cascade. It should be noted that a day-14 retrieval time point was often chosen to allow elucidation of earlier immune cell and cytokine signaling events as well as later stage myofibroblast recruitment and fibrosis deposition, which become much more robust by this point in time.

\section{Adaptive and innate immune cells in the fibrotic cascade}

To better understand the cellular components driving the fibrotic response, we examined 500 $\mu \mathrm{m}$ alginate spheres retrieved after 14 days, in a range of C57BL/6-derived strains with different immune mutations. A total of 7 strains were studied, ranging from fully immune competent wild type versus knockout (KO) strains with varying levels of immunodeficiency (Supplementary Table S1). Phase contrast imaging was used to examine relative levels of fibrotic overgrowth (Figure 3a and Supplemental Figures S5). Supporting the idea that B cell recruitment does not just correlate but may also contribute to simultaneous increases in aSMactin-expressing myofibroblasts (Figure 2c \& 2g), B cell loss (IghM ${ }^{\text {mull }}$, B KO) resulted in a partial loss of fibrosis (Figure 3a and Supplemental Figures S5 and S6). Additional T cell loss ( Rag2 $2^{\text {null }}, \mathrm{T} \& \mathrm{~B} \mathrm{KO}$ ) made fibrosis worse and more comparable to wild type (WT) levels (Figure 3a and Supplemental Figures S5e), perhaps due to the loss of the regulatory $\mathrm{T}$ cell subset important for suppressing overreaching immune reactions ${ }^{38}$. These findings suggest that some broad-spectrum anti-inflammatory agents may reduce anti-fibrotic activity, highlighting the need for more specific agents for drug-eluting systems. Ultimately, only with additional innate immune cell macrophage dysfunction in Rag2 $2^{\text {null }} / I 12 r \gamma^{\text {null }}$ (Rag2/ $\gamma$ KO) mice was a complete loss of implant-induced fibrosis observed (Figure 3a and Supplemental Figure S5d). Individual T cell (T KO, nude) and complement component C3 knockouts did not result in the loss of fibrosis (Supplemental Figure S5e \& S5f).

Western blot assays were performed on extracted proteins from retrieved spheres in all groups exhibiting significant decreases in fibrosis (B KO and $\mathrm{Rag} 2 / \gamma \mathrm{KO}$ ), as compared to wild type controls, to quantify changes in the fibrosis-associated myofibroblast marker aSMactin (Figure $3 \mathrm{~b} \& \mathrm{c}$ ). Expression results were further corroborated using qPCR analysis of RNA isolated from retrieved spheres, where aSMactin and Collagens 1a1 and 
1a2 showed similar significant decreases across both knockout models, as compared to WT (Figure 3d).

In addition to the study of spheres retrieved from the peritoneal space, which is relevant for cell encapsulation and transplantation ${ }^{21,23,27}$, alginate spheres were also implanted into the same wild type and knockout strains in the subcutaneous space (Figures $3 e$ \& 3f), more relevant for general biocompatibility assessment. Immune cell activity at the site of implantation was analyzed using Prosense, a fluorescent indicator of immune-associated inflammation ${ }^{39}$. IVIS imaging 7 days post-implantation showed decreases in alginateinduced inflammation in both B and Rag2/ $\gamma$ knock-out models (Figure 3e). After 1-month, subcutaneous spheres were excised and processed for histology (H\&E and Masson's Trichrome). In general, spheres were completely embedded and individually sequestered in WT mice, but only had an outer, thin fibrotic capsule in B KO mice. No fibrosis was observed in macrophage dysfunctional (Rag2/ $\gamma \mathrm{KO}$ ) mice (Figure $3 \mathrm{f}$ and Supplemental Figure S7). FACS analysis of retrieved capsules and surrounding tissue verified that wildtype subcutaneous immune responses are local to the implant (Supplemental Figure S8) and similar in composition to those in the IP space (Figure 3g). Lastly, FACS analysis showed a $\sim 60 \%$ decrease in macrophage and neutrophil presence on spheres taken from $B$ $\mathrm{KO}$ mice, and an essentially complete loss of adherent cells in macrophage dysfunctional (Rag2/ $\gamma \mathrm{KO}$ ) mice (Figure 3g).

\section{Macrophages, not neutrophils, are necessary for fibrosis}

Both macrophages and neutrophils were on the surface of alginate spheres even 1 day following implantation into wild type C57BL/6 mice (Figure 2c). One hypothesis as to why microspheres implanted into $\mathrm{Rag} 2 / \gamma \mathrm{KO}$ mice are fibrosis-free is that macrophages are necessary for fibrosis (Figure 3 ). However, Rag $2 / \gamma$ KO rodents still have macrophages, though they are reported to be reduced in number and dysfunctional, not maturing appropriately in response to cytokine stimulation ${ }^{40}$ (Supplementary Table S1). Since the Rag2/ $\gamma$ model also lacks adaptive $\mathrm{T}$ and $\mathrm{B}$ cells, innate immune natural killer cells, as well as functional dendritic cells, other unknown consequences affecting immune signaling and homeostasis may be possible. It has also recently been suggested that neutrophils are recruited first in response to protein deposition, with later macrophage response, in an initiated fibrotic cascade to implanted material devices ${ }^{15}$. Therefore, to further clarify the role of macrophages and neutrophils in fibrosis we induced depletion of macrophages, neutrophils, or both together by clodronate liposome (clodrosome) and/or targeted Ly6g (clone 1A8) neutrophil-depleting antibody, in implanted wild type C57BL/6 mice (Figure 4a and Supplemental S9). Depletion was started 3 days prior to implantation in all cases to ensure innate immune population ablation prior to alginate exposure. The extent and specificity of depletions were analyzed by FACS (Figure $4 \mathrm{~b} \& 4 \mathrm{c}$ and Supplemental Figures S10 and S11). Interestingly, fibrosis was only eliminated with targeted macrophage depletion, both alone and in combination with neutrophil removal (Figure 4a and Supplemental Figure S9). Neutrophil depletion alone, verified to be complete by FACS analysis (Supplemental Figure S11b, middle), appeared to cause alginate spheres to instead clump more, an effect seen with exacerbated immunity in the case of smaller diameter implants $^{27}$ (Figure 4a and Supplemental S9). This may be a consequence of the loss of the 
Ly6g-granulocyte myeloid derived suppressor cell (MDSC) subset, which, like $\mathrm{T}_{\text {regs, }}$, prevent excessive immune responses ${ }^{38}$. Prominent drug-elution systems to date have largely focused on the incorporated broad-spectrum anti-inflammatories ${ }^{24,31}$. However, these results suggest that targeted inhibition of a few select populations (innate immune macrophages and adaptive immune $B$ cells) while leaving all others intact (ie., immunosuppressive $T_{\text {regs }}$ and MDSCs) may lead to improved inhibition of FBR to implanted material devices.

Lastly, RNA samples (fibrosed capsules and attached, fibrosed epididymal/omental fat tissue) derived from all treatment groups (mock vs. alginate-implanted wild type, knockouts or depletions) were analyzed with a multiplexed NanoString array for all currently known mouse cytokines and cytokine receptors (Figure 4d-f and Supplemental Figure S12). Numerous genes, induced by alginate implantation, ( $2^{\text {nd }}$ versus $1^{\text {st }}$ column, mock), were eliminated upon macrophage depletion, both alone and in combination with neutrophil depletion ( $3^{\text {rd }}$ and $5^{\text {th }}$ columns). Many hits showing increased gene expression following implantation were corroborated by expression enrichment upon sorting macrophage cells away from the bulk mixed cellular response. Macrophage-specific genes exhibited an augmented increase in red, whereas non-macrophage genes showed dilution (either decreasing back to mock control levels (black), or below, green; $2^{\text {nd }}$ to last column). Using these metrics (lost upon depletion and increased upon sorting), macrophage-specific gene subsets were identified (Figures $4 \mathrm{~d} \& 4 \mathrm{e}$, partial). Genes lost upon macrophage depletion, but instead reduced upon sorting, were also identified (Figures 4e, partial, \& 4f). This subset is believed to be associated with cells (ie., B cells, Cd19) recruited downstream of macrophages in the fibrotic cascade. This hypothesis is driven by the observation that this gene subset is not macrophage-specific (due to lack of enrichment upon sorting), but still lost upon macrophage depletion. Thus, their expression is dependent on earlier macrophage activation and recruitment.

\section{CSF1R inhibition prevents host responses to implants}

While elimination of macrophages by clodrosome treatment can prevent fibrosis of hydrogel alginate (Figures 4a), it is unlikely this approach would be suitable for human use $\mathrm{e}^{41}$. An ideal anti-fibrotic drug therapy would only modulate and not eliminate this innate immune population, thus avoiding unnecessary immune suppression and resulting side effects. One macrophage-specific factor identified by our NanoString analysis was the cytokine receptor colony stimulating factor-1 receptor (CSF1R) (Figure 4d). This receptor has been previously reported to play a role in selectively polarizing and modulating macrophage phenotypes in cancer $^{42}$. To test whether inhibition of this receptor could prevent macrophage-dependent biomaterial fibrosis, we examined the potential of the inhibitory small molecule GW2580 to inhibit the kinase domain thus preventing cross-phosphorylation and activation of CSF1 $\mathrm{R}^{43}$ and the fibrotic response to implanted microspheres. Treatment of C57BL/6 mice with GW2580 prevented the fibrotic response to a range of implanted materials, including 500 $\mu \mathrm{m}$ alginate as well as $500 \mu \mathrm{m}$ ceramic glass and polymer polystyrene spheres, all implanted for 14 days intraperitoneally (Figures 5a-d and Supplemental Figures S13-S15). CSF1R inhibition by GW2580 was as effective as clodrosome-based macrophage depletion at inhibiting host immune attack to alginate, glass and polystyrene spheres (Figure 4c vs. 5b, and 5d), showing that a targeted and selective inhibition of macrophages, rather than 
depletion, is sufficient for inhibiting the FBR to multiple classes of material implants. Uninhibited fibrotic responses against all three materials importantly share the same core immune markers, and cytokine and cytokine receptor signaling, including CSF1R (Figure 5e and Supplemental Figure S16). And, as mentioned earlier, this response is dependent upon the implanted material, and not endotoxin, as analysis did not detect the presence of general pyrogens, endotoxins or glucan (Supplementary Table S2).

After establishing efficacy of this treatment strategy, we decided to also analyze the gene expression patterns from alginate spheres retrieved from these mice. This was done to determine which of the earlier identified macrophage-specific genes would or would not be affected by CSF1R inhibition (Figures 4d vs. 4e). Specifically, we inspected all macrophageassociating genes that were both significantly increased 2 weeks following implantation into the intraperitoneal space ( $W T$ vs Mock controls), as well as eliminated or significantly decreased following macrophage depletion. Interestingly, the presence of many macrophagespecific factors not affected by CSF1R inhibition (Figure 4e) suggests possible spared macrophage function, not observed with more blunt macrophage depletion and removal. VEGF protein production, directly associated with macrophage repair function ${ }^{44}$, was significantly diminished in both macrophage depletion groups, but not significantly affected with GW2580 treatment (Supplemental Figure S17). Furthermore, while macrophage phenotype changed with daily GW2580 treatment, no effect was observed on intraperitoneal cell counts or other macrophage-associated functions, including skin incision wound healing, macrophage phagocytic activity, and reactive oxygen species production (Supplemental Figure S18). While no functional changes were observed in these metrics, the lack of macrophage attachment, activation, and fusion as well as later fibrosis demonstrates a change in phenotype and function. It is possible that phagocytosis and $\mathrm{NO}$ production are unrelated to these activities. We note that gene expression of NOS2 is not engaged in the implantation response to alginate (Supplemental Figure S12, right column, $3^{\text {rd }}$ gene down from the top). Treatment with the CSF1R inhibitor GW2580 appears to divert the immune response to foster a pro-healing rather than fibrotic outcome. The differential effect of CSF1R inhibition on these subsets of genes (Figure $4 \mathrm{~d}$ and $\mathrm{f} v \mathrm{vs}$. $4 \mathrm{e}$ ) indicates that certain pathways within these cells are in fact being affected. For instance, inhibited Cx3cr1 and IL21r expression suggests improved healing yet reduced fibrotic capacity ${ }^{45}$, 46 . However, more experimentation will be needed to fully examine such cellular mechanisms.

\section{Macrophages recruit fibrosis-potentiating B cells via CXCL13}

The chemokine CXCL13 has been shown to be expressed by monocytes/macrophages and is reported to be responsible for B cell recruitment in a model of lymphoid neogenesis ${ }^{47}$. Interestingly, CXCL13 was also identified by gene expression profiling of tissue following implantation of alginate microspheres (Figure 2d and Supplemental Figure S4c), as well as lost upon macrophage depletion (Figure 4d). CXCL13 was one of numerous immune markers and cytokines that either disappeared or was decreased in expression ( $\sim 5$ to 10 -fold) below background levels following macrophage depletion and reduced (back to background levels) following CSF1R inhibition with GW2580 (Figure 4d). CXCL13 was also enriched upon sorting the macrophage population away from the bulk heterogeneous host cell infiltrate. To determine whether it plays a role in B cell recruitment in the observed 
biomaterial-induced fibrotic cascade, neutralizing antibody was administered to alginateimplanted wild type C57BL/6 mice. CXCL13 neutralization resulted in loss of B cell recruitment and reduced fibrosis (Figures 5a, 5f, and Supplemental Figure S19), similar to levels observed with $\mathrm{B}$ cell knock out (Figures $5 \mathrm{~g}$ and $3, B \mathrm{KO}$ ). These findings suggest a role for $\mathrm{B}$ cells in potentiating fibrosis, perhaps due to their ability to regulate macrophage phenotype and response ${ }^{48}$. While $\mathrm{B}$ cells have been designated as an adaptive immune cell throughout this study, it is possible that they affect fibrosis in a manner generally associated with the innate immune response by secreting cytokines rather than antibodies. Their exact role will need to be investigated further. Interestingly, B cell loss was also observed with clodrosome macrophage depletion and CSF1R inhibition (Figures 4b, 4c, 4f, and 5b), once again suggesting that macrophages may have a role in their downstream recruitment. NanoString and western blot analysis for CXCL13 over 1, 4, 7, 14, and 28-day alginate microsphere implantation showed detectable RNA and protein levels by day 4 onward (Figure 2d and Supplement Figure S4c), matching observed kinetics for maturation of macrophages from immature monocytes (Figure 2c), and just preceding apparent day 7 B cell recruitment (Figures $2 \mathrm{~b} \& 2 \mathrm{~g}$ ). Taken together, these results indicate that macrophages are required and responsible for downstream $\mathrm{B}$ cell recruitment via the $\mathrm{B}$ cell chemoattractant CXCL13, and that, upon arrival, these B cells enhance fibrotic deposition. Highlighting the translational significance of these findings, CXCL13 was recently shown in human patients to be a biomarker for idiopathic pulmonary fibrosis ${ }^{49}$.

\section{Immune responses to implanted biomaterials in non-human primates}

To study whether these findings were also observed in higher order non-human primates (NHPs), we implanted $500 \mu \mathrm{m}$ diameter SLG20 alginate spheres, either intraperitoneally (N $=2$ ) or subcutaneously into the dorsal regions $(\mathrm{N}=4)$ of cynomolgus monkeys for 28 days. At 28 days post-implantation the $500 \mu \mathrm{m}$ SLG20 alginate spheres were heavily embedded in host omental or subcutaneous tissues (Figure $6 \mathrm{a} \mathrm{and}^{27}$ ). Excised tissue obtained from the IPimplanted $500 \mu \mathrm{m}$ SLG20 alginate spheres or non-fibrosed ("mock") control tissue were examined through histological analysis with H\&E and Masson's Trichrome staining (Figure $6 \mathrm{~b}$ and Supplemental Figure S20a-b). Extensive embedding and fibrosis buildup (up to 100 $\mu \mathrm{m}$ thick), similar to that seen in C57BL/6 mice, is visible. Confocal imaging of sections taken from retrieved 28-day IP omentum tissue confirmed extensive macrophage cellular deposition and fibrosis-associated alpha Smooth Muscle actin-positive, activated myofibroblast coverage (Figure 6c and Supplemental Figure S20c-e). Since we do not have earlier day 14 staining in the primates as we do in the C57BL/6 mice, it remains unclear whether these observed myofibroblasts levels are already lower at day 28 . With that said, there are areas of lower aSMactin stain around embedded capsules (Supplemental Figure S20c-e), which could indicate partial resolution. Furthermore, histology shows the emergence of fused foreign body giant cells (FBGCs) immediately surrounding embedded capsules (Figure 6b and Supplemental Figure S20a-b). Importantly, upon staining for CSF1R (Figure 6c, inset, and Supplemental Figure S20c-e), we observed that both infiltrating macrophages and FBGCs adjacent to the implanted material have very high CSF1R expression ${ }^{50}$, indicating that both of these populations may be affected by GW2580 treatment. Innate immune responses (macrophage and myeloid/neutrophil) were determined 
by FACS to be similar between C57BL/6 mice and NHP cynomolgus monkeys (Figure 6d). Lastly, to confirm that the immune factors identified in the C57BL/6 model are also relevant for both intraperitoneal as well as subcutaneous alginate implants isolated previously ${ }^{27}$ from NHPs, NanoString analysis was performed on RNA derived from excised fibrotic alginate implants. Results showed increased expression of numerous factors common to the C57BL/6 model, including multiple cytokine receptors and cytokines, such as CSF1R and CXCL13, and innate immune markers, such as CD68 and CD66b (in place of Ly6g/Gr1, which does not exist in NHPs and humans) (Figure 6e). Together, these findings support the use of the C57BL/6 mouse model to recapitulate the FBR seen in higher species such as non-human primate cynomolgus monkeys and human patients. The fact that both models share similar immunologic responses, not just in content but also kinetics and magnitude, adds additional credence to their use for research validation before translation into the clinic.

In summary, we have identified, through targeted perturbation/depletion studies, essential innate (macrophage) and adaptive (B cell) immune populations in the fibrotic response to implanted materials. Serial perturbation samples in conjunction with a complete known cytokine and cytokine receptor array were utilized to identify additional essential components of the fibrotic cascade, and evaluated to determine potential drug therapies to inhibit material implant-induced fibrosis. The host rejection responses and immunobiology were similar in both C57BL/6 mice and cynomolgus monkeys. While the chemokine CXCL13 was implicated in downstream recruitment of B cells, its blockade only resulted in a partial loss of fibrosis. Surprisingly, inhibition of the macrophage-associated cytokine receptor CSF1R eliminated host immune-mediated recognition and propagation of foreign body rejection responses to a broad spectrum of materials encompassing hydrogels, ceramics, and plastics. Importantly, as opposed to more blunt macrophage depletion, specific CSF1R inhibition spared other essential macrophage function. We believe these findings provide insight into the immunological basis of FBR, and may allow for the development of macrophage-specific agents for integration into already existing and prominently used drugelution systems to reduce the fibrotic response to implanted medical devices.

\section{METHODS}

\section{Materials and Reagents}

All in vitro reagents were obtained from Life Technologies (Carlsbad, CA), unless otherwise noted. Antibodies: Alexa Fluor-conjugated anti-mouse CD68, Ly-6G/Ly-6C (Gr-1), CD11b, CD19, and IgM (described below) were purchased from BioLegend Inc. (San Diego, CA). For primate immunostaining, anti-human CD68 Alexa Fluor-conjugated antibody was purchased from Santa Cruz (Dallas, TX). The same CD11b (anti-mouse/human) antibody (BioLegend) was used for both primate and mouse staining. Cy3-conjugated anti-mouse alpha smooth muscle actin antibody and glass spheres (acid washed) of medium size (conformed to 425-600 $\mu \mathrm{m}, 30-40$ U.S. sieve) (Cat\#: G8772, Lot\#: SLBL7288V) were purchased from Sigma Aldrich (St. Louis, MO). Polystyrene spheres of medium (mean $526.6 \mu \mathrm{m}+/-53.3 \mu \mathrm{m}$ ) size (Cat\#:136, Lot\#: 30055$)$ were purchased from Phosphorex (Hopkinton, MA). A sampling of materials used in this study were submitted for endotoxin testing by a commercial vendor (Charles River, Wilmington, MA) and the results showed 
that spheres contained $<0.05 \mathrm{EU} / \mathrm{ml}$ of endotoxin levels (below detectable limits) (Supplemental Table S2).

\section{Fabrication of alginate hydrogel spheres}

Alginate hydrogel spheres were made with an in-house customized electro-jetting system: voltage generator, vertical syringe pump (Harvard Apparatus), and a gelation bath basin. Voltage was coupled to the syringe needle dispensing the alginate and grounded to the gelling bath vessel. Spheres were made with a $1.4 \%$ solution of commercially available sterile alginate (PRONOVA SLG20, NovaMatrix, Sandvika, Norway) dissolved in $0.9 \%$ saline $(\mathrm{pH} \approx 7.4$, Osmotic pressure $\approx 290 \mathrm{mOsm}$ ), and crosslinked with $250 \mathrm{~mL}$ of sterile $\mathrm{BaCl}_{2}$ gelling solution (20mM BaCl $2,250 \mathrm{mM}$ D-Mannitol, 25mM HEPES, $\mathrm{pH} \approx 7.4$, Osmotic pressure $\approx 290 \mathrm{mOsm}$ ). Alginate hydrogel $500 \mu \mathrm{m}$ diameter microspheres were generated with a $25 \mathrm{G}$ blunt needle, a voltage of $5 \mathrm{kV}$ and a $200 \mu \mathrm{l} / \mathrm{min}$ flow rate. Immediately after gelation, alginate spheres were washed with HEPES buffer $(25 \mathrm{mM}$ HEPES, $1.2 \mathrm{mM} \mathrm{MgCl}_{2} \times 6 \mathrm{H} 2 \mathrm{O}, 4.7 \mathrm{mM} \mathrm{KCl}, 132 \mathrm{mM} \mathrm{NaCl}_{2}, \mathrm{pH} \approx 7.4$, $\approx 290$ mOsm) 4 times and stored overnight at $4{ }^{\circ} \mathrm{C}$. Immediately prior to implantation, spheres were washed an additional 2 times with $0.9 \%$ saline. A sampling of the fabricated hydrogels was submitted for endotoxin testing by a commercial vendor (Charles River, Wilmington, MA) and the results showed that SLG20 hydrogels contained $<0.05 \mathrm{EU} / \mathrm{ml}$ of endotoxin levels (below detectable limits).

\section{Implantation surgeries}

All protocols were approved by the MIT Committee on Animal Care, and all surgical procedures and post-operative care were supervised by MIT Division of Comparative Medicine veterinary staff. All mice, wild type male immune-competent (non-diabetic), STZinduced diabetic, as well as knockout C57BL/6 mice, were ordered pathogen-free from Jackson Laboratory (Bar Harbor, ME) or Taconic (Hudson, NY). The status of all mice was subsequently verified by testing sentinel animals, shown to be negative for at least 12 known mouse pathogens. Implanted mice were anesthetized with 3\% isoflurane in oxygen and had their abdomens shaved and sterilized using betadine and isopropanol. C57BL/6-Nude (T KO, B6NU), C57BL/6-Rag2 ${ }^{\text {null }}$ (T \& B KO, RAGN12), C57BL/6-Rag2 ${ }^{\text {null }} / \mathrm{Il} 2 \mathrm{r} \gamma^{\text {null }}$ (Rag2/ $\gamma, 4111$ ) were ordered from Taconic (Hudson, NY), and C57BL/6-IghM ${ }^{\text {null }}$ (BKO, 002288), (C57BL/6) B6.129S6-Ccr6tm1(EGFP)Irw/J. (Ccr6, 013061), and C57BL/6-C3 KO (C3 KO, 003641) mice were ordered from Jackson Laboratory, Bar Harbor, ME). Preoperatively, all mice received $0.05 \mathrm{mg} / \mathrm{kg}$ buprenorphine and $0.2 \mathrm{~mL}$ of $0.9 \%$ saline subcutaneously for presurgery analgesia and dehydration prevention. A midline (abdomen) incision $(0.5 \mathrm{~mm})$ was made and the peritoneal lining was exposed using blunt dissection. The peritoneal wall was then grasped with forceps and a $0.5-1 \mathrm{~mm}$ incision was made along the linea alba. A desired volume of spheres (all materials) were then loaded into a sterile pipette and implanted into the peritoneal cavity. The incision was then closed using 5-0 taper-tipped polydioxanone (PDS II) absorbable sutures, and the skin was closed using a wound clip and VetBond tissue glue. For subcutaneous implantation, $\sim 200-300 \mu \mathrm{L}$ of $500 \mu \mathrm{m}$ SLG20 spheres were injected $\mathrm{SC}$ following anesthesia with isofluorane. All primate implant samples were derived by excising fibrosed, material-containing omentum and subcutaneous tissues 28 days following implantation of SLG20 $500 \mu \mathrm{m}$ diameter spheres in cynomolgus macaques, as described ${ }^{27}$. 
For targeted macrophage depletions, clodrosome ( $200 \mu \mathrm{L} /$ mouse) (Encapsula Nano Sciences, Nashville, TN) were injected intraperitoneally starting at -3 days (prior to implantation, day 0 ), and for every 7 days thereafter (so days $-3,4$, and 11 ). To achieve neutrophil depletion, affinity purified anti-Ly6g (Clone 1A8) antibody (250 ug/mouse) (BioLegend, San Diego, CA) was administered also starting at day -3 , and every 3 days thereafter (except the day of implantation). For the combination therapy, in order to avoid drug-antibody interactions, each agent was administered 4 hours apart from one another. To neutralize secreted CXCL13, anti-CXCL13 antibody (mAB470, R\&D Systems) was injected IP in sterile $1 \mathrm{X}$ PBS at a dose of $100 \mu \mathrm{g} /$ mouse, once every 3 days, starting 3 days prior to implantation as well. For selective macrophage inhibition and polarization, the CSF1Rtargeted inhibitor GW2580 (LC Labs, Woburn, MA), was dissolved in a 1:1 DMSO/PEG400 solution, and injected daily (starting 1 day prior to implantation) at a dose of $160 \mathrm{mg} / \mathrm{kg} \mathrm{BW}$ subcutaneously (SC), to eliminate concerns of vehicle directly perturbing responding immune cells as well as a recovering implant site.

\section{IVIS imaging}

C57BL/6 mice (6-8 weeks old) were utilized for this assay. 200-300 $\mu$ of alginate spheres were resuspended in saline, and injected subcutaneously into the mouse on both left and right sides of upper back. The mice were shaved to get rid of the hair on the entire back before injection, and fed on sterilized AIN-93G purified rodent diet (TD 94045, Harlan) to minimize the fluorescent background after injection. Six days later, $100 \mu \mathrm{l}(4 \mathrm{nmol})$ of ProSence 750 FAST (NEV11171, PerkinElmer Inc.) per mouse was injected intravenously via tail vein. At day 7 (24 hours post intravenous administration of the ProSense 750 FAST substrate, cleaved into a fluorescent tracking product by immune secreted cathepsin enzymes), the mice were scanned by IVIS Spectrum system (Xenogen, Caliper LifeScience). The mice were anesthetized using $3 \%$ isofluorane in oxygen and maintained at the same rate throughout the procedure, the new grown hair were removed by Nair hair removal lotion, and then the mice were scanned by the IVIS Spectrum system at the settings of Exposure=7.50, Binning=Medium, FStop=2, Excitation $=605 \sim 640 \mathrm{~nm}$ and Emission=660 760nm. The images were analyzed with LivingImage Software, and the duplicated ROI of lower back on the same mouse was used as control during the signal quantification.

\section{Retrieval of cells, tissues, and materials}

At desired time points post-implantation, as specified in figures, mice were euthanized by $\mathrm{CO}_{2}$ administration, followed by cervical dislocation. In certain instances, $5 \mathrm{ml}$ of ice cold PBS was first injected in order perform an intraperitoneal lavage to rinse out and collect free-floating intraperitoneal immune cells. An incision was then made using forceps and scissors along the abdomen, and intraperitoneal lavage volumes were pipetted out into fresh $15 \mathrm{ml}$ falcon tubes (each prepared with $5 \mathrm{ml}$ of RPMI cell culture media). Next, a wash bottle tip was inserted into the abdominal cavity. KREBS buffer was then used to wash out all material spheres into petri dishes for collection. After ensuring all the spheres were washed out or manually retrieved (if fibrosed directly to intraperitoneal tissues, in particular epididymal and omental fat pads), they were transferred into $50 \mathrm{~mL}$ conical tubes for downstream processing and imaging. In certain instances, after intraperitoneal lavage, 
portions of fibrosed intraperitoneal tissues and material spheres were also excised for downstream FACS and expression analyses.

\section{Imaging of the retrieved material spheres}

For phase contrast imaging retrieved materials were gently washed using Krebs buffer and transferred into $35 \mathrm{~mm}$ petri dishes for phase contrast microscopy using an Evos Xl microscope (Advanced Microscopy Group). For bright-field imaging of retrieved materials, samples were gently washed using Krebs buffer and transferred into $35 \mathrm{~mm}$ petri dishes for bright-field imaging using a Leica Stereoscopic microscope.

\section{Confocal Immunofluorescence}

Immunofluorescence imaging was used to determine immune populations attached to spheres. Materials were retrieved from mice and fixed overnight using $4 \%$ paraformaldehyde at $4{ }^{\circ} \mathrm{C}$. Samples were then washed twice with KREBS buffer, permeabilized for $30 \mathrm{~min}$ using a $0.1 \%$ Triton X100 solution, and subsequently blocked for 1 hour using a $1 \%$ bovine serum albumin (BSA) solution. Next, the spheres were incubated for 1 hour in an immunostaining cocktail solution consisting of DAPI $(500 \mathrm{nM})$, specific marker probes (1:200 dilution) in BSA. After staining, spheres were washed three times with a $0.1 \%$ Tween 20 solution and maintained in a 50\% glycerol solution. Spheres were then transferred to glass bottom dishes and imaged using an LSM 700 point scanning confocal microscope (Carl Zeiss Microscopy, Jena Germany) equipped with 5 and 10X objectives. Obtained images were adjusted linearly for presentation using Photoshop (Adobe Inc. Seattle, WA). For antigen-specific immunostaining, non-human primate (cynomolgus macaque) intraperitoneal sphere-embedded omenta were sectioned and stained according to traditional antigen retrieval and immunofluorescent methods, specifically looking at cellular nuclei (DAPI), macrophage marker CD68-AF488 (Santa Cruz, CA) and Cy3-conjugated antimouse alpha smooth muscle actin (fibrosis-associated myofibroblast marker) (Sigma Aldrich, St. Louis, MO). Anti-human CSF1R-AF488 (BioLegend) was also used.

\section{Histological processing for H\&E and Masson's Trichrome staining}

Retrieved material-containing tissue (omentum and/or subcutaneous) was fixed overnight using $4 \%$ paraformaldehyde at $4{ }^{\circ} \mathrm{C}$. After fixation, alginate sphere or retrieved tissue samples were washed using $70 \%$ alcohol. The materials were then paraffin embedded, sectioned and stained according to either standard histological (H\&E or Masson's Trichrome) or antigen-specific methods (as described above).

\section{Western Blotting}

Protein was extracted directly from materials for western blot analysis. Retrieved materials were prepared by lysis of covering cellular overgrowth in Pierce RIPA buffer (Cat. \#89901, Thermo Scientific) with protease inhibitors (Halt Protease inhibitor single-use cocktail, Cat. \#78430, Thermo Scientific) on ice, and then lysed by sonication (for 30 seconds on, 30 seconds off, twice at $70 \%$ amplitude). Samples were then agitated constantly for 2 hours at $4^{\circ} \mathrm{C}$. Lysates were centrifuged for $20 \mathrm{~min}$ at $12,000 \mathrm{rpm}$ at $4^{\circ} \mathrm{C}$, and protein-containing supernatants were collected in fresh tubes, on ice. In samples from fatty tissue, an excess of 
fat (a top layer on the supernatant) was removed before supernatant transfer. $20 \mu \mathrm{g}$ protein (quantified by BCA assay, Pierce BCA protein assay kit, Cat. \#23225, Thermo Scientific) for each lane was boiled at $95^{\circ} \mathrm{C}$ for $5 \mathrm{~min}$ and electrophoresed on SDS-polyacrylamide gels (Any kD 15-well comb mini-gel, BioRad, Cat. \#456-9036) and then blotted onto nitrocellulose membranes (BioRad, Cat. \#162-0213). Blots were probed with anti-alpha Smooth Muscle actin antibody (1:400 dilution, Rabbit polyclonal to alpha smooth muscle actin; Cat. \#ab5694, AbCam) and anti- $\beta$-actin antibody (1:4000 dilution, monoclonal anti- $\beta$ actin antibody produced in mouse; Cat. \#A1978, Sigma Aldrich) as a loading control followed by donkey anti-rabbit (1:15,000 dilution, Cat. \#926-32213, Li-Cor) and goat antimouse (1:15,000 dilution, Cat. \#926-68070, Li-Cor) fluor-conjugated secondary antibodies. Bands were visualized using an Odyssey detector (Li-Cor, Serial No. ODY-2329) at 700 and $800 \mathrm{~nm}$ wavelengths. For CXCL13 detection, a rabbit anti-CXCL13/BCA1 polyclonal antibody (Bioss, Woburn, MA) was used.

\section{qPCR analysis}

Total RNA was isolated from tissue (adjacent epididymal and omental fat pad tissue alone for non-implant and mock (saline injected) controls, or fibrosed spheres with adhered tissue and immune overgrowth, if present), liquid nitrogen snap-frozen immediately following excision, using TRIzol (Invitrogen, Carlsbad, CA) according to the manufacturer's instructions. In addition, to help ensure complete tissue disruption, we also employed strong mechanical disruption with a Polytron homogenizer. Thus, gene expression signatures shown throughout are proportional and representative of the entire cell population present on and/or around retrieved materials. Before reverse transcription using the High Capacity cDNA Reverse Transcription kit (Cat. \#4368814; Applied Biosystems, Foster City, CA), all samples were first normalized for comparison by loading the same input $1 \mu \mathrm{g}$ total RNA in a volume of $20 \mu \mathrm{l}$ for each sample. cDNA (4.8 $\mu \mathrm{l} ; 1$ :20 dilution) in a total volume of $16 \mu \mathrm{l}$ (including SYBR Green and PCR primers) was amplified by qPCR with the following primers. Primers (Supplemental Table S3) were designed using Primer Express software (Applied Biosystems, Carlsbad, CA, USA) and evaluated using LaserGene software (DNAStar, Madison, WI, USA) to ensure either mouse (host)-specificity. Samples were incubated at $95^{\circ} \mathrm{C}$ for $10 \mathrm{~min}$ followed by 40 cycles of $95^{\circ} \mathrm{C}$ for $15 \mathrm{sec}$ and $60^{\circ} \mathrm{C}$ for $1 \mathrm{~min}$ in an ABI PRISM 7900HT Sequence Detection System (Applied Biosystems). Results were analyzed using the comparative $\mathrm{C}_{\mathrm{T}}\left(\Delta \Delta \mathrm{C}_{\mathrm{T}}\right)$ method and are presented as relative RNA levels, as compared to control cell samples as specified in figure legends after normalization to the $\beta$-actin RNA content of each sample. To further ensure proper normalization and sample handling across multiple retrieval time points, RNA for all samples for each harvest condition (ie., IP lavage, spheres with or without adhered cells and fibrosis, and adjacent omental and/or epididymal tissues with infiltration, as described), were quantified, reverse transcribed, and analyzed by qPCR in parallel.

\section{Luminex multiplexed cytokine and ELISA analyses}

Cytokine array analysis was performed using the Luminex Bio-Rad Bio-Plex Pro mouse cytokine panel based on Luminex beads (multiplexing both the 23 and 8-cytokine arrays) (Bio-Rad, Hercules, CA), according to manufacturer's instructions. 1:4 diluted mouse sera samples were analyzed for the time course, and protein lysates taken from fibrosed tissue 
and alginate microspheres, loaded at a concentration of $500 \mu \mathrm{g} / \mathrm{mL}$ (200 uL per sample), were run to compare cytokine responses across mock (saline) treated versus wildtype, knockout or serially perturbed/depleted C57BL/6 mice implanted with $500 \mu \mathrm{m}$ SLG20 alginate spheres. Samples were added to the panel of beads bearing capture antibodies for the analytes of interest, and agitated for 30 minutes. Sample plates were subsequently washed using an automated magnetic plate washer (Bio-Rad), and a biotinylated detection antibody for each of the 32 cytokines was added followed by agitation for 30 minutes. After another wash, streptavidin-PE was added, the plate was agitated for 10 minutes, washed, and resuspended in assay buffer. The plate was then read on a BioPlex-200 plate reader, with absolute concentrations obtained through fitting a standard curve. For Luminex runs, protein lysates were prepared using an NP-40-based lysis buffer, as opposed to the SDS-based RIPA buffer used for western blotting (described above) to maintain native state protein folding. For mouse IgM analysis, an ELISA from eBioscience (San Diego, CA) was used, according to manufacturer's specifications, with both sera and protein lysates for IgM.

\section{FACS analysis}

Single-cell suspensions of freshly excised tissues were prepared using a gentleMACS Dissociator (Miltenyi Biotec, Auburn, CA) according to the manufacturer's protocol. Singlecell suspensions were prepared in a passive PEB dissociation buffer (1X PBS, pH 7.2, 0.5\% BSA, and $2 \mathrm{mM}$ EDTA) and suspensions were passed through $70 \mu \mathrm{m}$ filters (Cat. \#22363548, Fisher Scientific, Pittsburgh, PA). This process removed the majority of cells adhered to the surface ( $>90 \%$ ) (See Supplemental Figure $17 \mathrm{from}^{27}$ ). All tissue and material sample-derived, single-cell populations were then subjected to red blood cell lysis with $5 \mathrm{ml}$ of 1X RBC lysis buffer (Cat. \#00-4333, eBioscience, San Diego, CA, USA) for $5 \mathrm{~min}$ at $4^{\circ} \mathrm{C}$. The reaction was terminated by the addition of $20 \mathrm{ml}$ of sterile $1 \mathrm{X}$ PBS. The cells remaining were centrifuged at $300-400 \mathrm{~g}$ at $4^{\circ} \mathrm{C}$ and resuspended in a minimal volume $(\sim 50$ $\mu \mathrm{l}$ ) of eBioscience Staining Buffer (cat. \#00-4222) for antibody incubation. All samples were then co-stained in the dark for $25 \mathrm{~min}$ at $4{ }^{\circ} \mathrm{C}$ with two of the fluorescently tagged monoclonal antibodies specific for the cell markers CD68 (1 $\mu \mathrm{l}(0.5 \mu \mathrm{g})$ per sample; CD68Alexa647, Clone FA-11, Cat. \#11-5931, BioLegend), Ly-6G (Gr-1) (1 $\mu \mathrm{l}(0.5 \mu \mathrm{g})$ per sample; Ly-6G-Alexa-647, Clone RB6-8C5, Cat. \#108418, BioLegend), CD11b (1 $\mu \mathrm{l}$ (0.2 $\mu \mathrm{g})$ per sample; or CD11b-Alexa-488, Clone M1/70, Cat. \#101217, BioLegend). For alpha smooth muscle actin (fibrosis) analysis, additional cell aliquots were also fixed in $1 \%$ paraformaldehyde and permeabilized with $0.1 \%$ triton X-100 before being stained with Cy3conjugated anti-mouse aSMactin antibody (1:100) (Sigma Aldrich, St. Louis, MO). Two ml of eBioscience Flow Cytometry Staining Buffer (Cat. \#00-4222, eBioscience) was then added, and the samples were centrifuged at $400-500 \mathrm{~g}$ for $5 \mathrm{~min}$ at $4^{\circ} \mathrm{C}$. Supernatants were removed by aspiration, and this wash step was repeated two more times with staining buffer. Following the third wash, each sample was resuspended in $500 \mu$ of Flow Cytometry Staining Buffer and run through a $40 \mu \mathrm{m}$ filter (Cat. \#22363547, Fisher Scientific) for eventual FACS analysis using a BD FACSCalibur (cat. \#342975), BD Biosciences, San Jose, CA, USA). For proper background and laser intensity settings, unstained, single antibody, and IgG (labled with either Alexa-488 or Alexa-647, BioLegend) controls were also run. For primate cell staining, anti-human CD68 Alexa Fluor-647-conjugated antibody (Clone KP1, 
Cat. \#sc-20060, Santa Cruz, Dallas, TX) was used in conjunction with the above BioLegend (anti-mouse/human) CD11b-AF488 antibody.

\section{Intravital Imaging - Ccr6-EGFP mice}

For intravital imaging, $500 \mu \mathrm{m}$ SLG20 hydrogel spheres were loaded with Qdot 605 (Life technologies, Grand Island, NY) and surgically implanted into (C57BL/6) B6.129S6Ccr6tm1(EGFP)Irw/J (Ccr6, 013061, Jackson Laboratory, Bar Harbor, ME) mice, as described above. After 14 days post implantation, the mice were placed under isoflurane anesthesia throughout and a small incision was made at the site of the original surgery to expose beads. The mice were placed on an inverted microscope and imaged using a $25 \mathrm{x}$, N.A. 1.05 objective on an Olympus FVB-1000 MP multiphoton microscope at an excitation wavelength of $860 \mathrm{~nm}$. While Ccr6 can show EGFP in adaptive T and B cells as well as innate immune dendritic cells (DCs), only B cells respond in our alginate material model. We previously showed macrophage responses via intravital imaging to implanted capsules ${ }^{27}$.

\section{NanoString analysis}

RNAs for mock-implanted (saline) treated controls, or for $500 \mu \mathrm{m}$ alginate sphere-bearing C57BL/6 mouse strains ( $\mathrm{n}=5 /$ group), including wild type, knockouts, and serially immune depleted wild type mice, were isolated from tissue samples taken at various time points after implantation, as described. For each of the knockout strains, they were normalized to their own (strain-specific) mock (saline) implanted controls, in order to eliminate any artifacts due to shifts in immune homeostasis, since gene knockouts were also present throughout development. Furthermore, to corroborate macrophage-specific changes, macrophages were also sorted from implanted wild type C57BL/6 mice, and also plotted relative to expression levels from the entire mock tissue cell population. Thus, macrophage-specific genes show enriched (red, increased) expression fold changes, and macrophage-independent gene show diluted (green, decreased) expression fold changes. In general, respective RNAs were quantified, normalized to the appropriate loading concentration $(100 \mathrm{ng} / \mu \mathrm{l})$, and then $500 \mathrm{ng}$ of each sample was processed according to NanoString manufacturer protocols for expression analysis via our customized multiplexed total (known) mouse cytokine and cytokine receptor expression panel, used for both immune strain comparisons with alginate, and material class comparisons just the wild type C57BL/6 strain. Another non-human primate custom panel was also used to corroborate gene hits in our cynomolgus macaque intraperitoneal and subcutaneous models. RNA levels (absolute copy numbers) were obtained following nCounter (NanoString Technologies Inc., Seattle, WA) quantification, and group samples were analyzed using nSolver analysis software (NanoString Technologies Inc., Seattle, WA).

\section{Macrophage functional testing}

For in vivo wound healing: C57BL/6 mice were anesthetized (same as above) and shaved, and skin incisions $1.5 \mathrm{~cm}$ in length were made along the midline. Each incision was then wound clipped shut for both vehicle and daily GW2580-treated C57BL/6 mice. Wound clips were removed and then replaced each imaging day up until day 7 (required to maintain them up until at least this time), after which clips were left off completely. Photos were acquired on days $0,1,4,7,10$, and 14 post-incision. After 14 days, mice were sacrificed, and skin 
samples were prepared for histological assessment (H\&E and Masson's Trichrome) of both vehicle and GW2580 treatment groups. Cross-sections through the incision line were generated to assess the level of in vivo healing following a 14-day treatment regimen.

For IP innate immune cell counts, FACS, and phagocytosis assays: cells were isolated by peritoneal lavage with $8 \mathrm{~mL}$ DPBS, diluted into RPMI, and then subsequently counted, and aliquoted either for staining and FACS analysis (as described above), or for phagocytosis assays. Peritoneal exudate macrophages isolated by IP lavage from $(n=5)$ mice in each treatment group were immediately plated $(400,000$ cells/well in a 24 -well plate) and incubated with fluorospheres for 90 minutes to determine phagocytic activity. $1 \mu \mathrm{m}$ orange fluorospheres (540/560) (Cat\# F13082, Invitrogen, Carlsbad, CA) were added to each well in a volume of $500 \mu \mathrm{L}$ from an initial dilution of $20 \mu \mathrm{L}$ into $10 \mathrm{~mL}$. After addition, plates were spun down at 1,000 rpm for $1 \mathrm{~min}$, and then placed in an incubator for 90 minutes to allow IP macrophages time to phagocytose the $1 \mu \mathrm{m}$ diameter fluorospheres. Following the 90-minute incubation, all wells were fixed with $4 \%$ paraformaldehyde and stored at $4 \mathrm{C}$ prior to imaging using a fluorescent and brightfield-capable EVOS microscope (Advanced Microscopy Group).

For ROS activity assessment: protein lysates were prepared from alginate spheres retrieved 14 days after IP implantation into untreated, vehicle-treated, and daily GW2580-treated C57BL/6 mice. $50 \mu \mathrm{g}$ of each lysate were aliquoted twice and incubated for $30 \mathrm{~min}$. at 37C with two different reactive oxygen specie (ROS) substrate solutions: $10 \mu \mathrm{M}$ APF (Cat\# A36003, Invitrogen, Carlsbad, CA) and $5 \mu$ M CellROX (Cat\# C10422, Invitrogen, Carlsbad, CA) diluted in PBS. Following appropriate incubation times, samples were read in a blackwall 96-well plate with a Tecan fluorescent-capable Infinite M1000 plate reader.

\section{Statistical analysis}

Data are expressed as mean \pm SEM, and $n=5$ mice per time point and per treatment group. These sample sizes were chosen based on previous literature. All animals were included in analyses except in instances of unforeseen sickness or morbidity. Animal cohorts were randomly selected. Investigators were not blind to performed experiments. For qPCR, western blot and wound healing quantification, IVIS imaging, or FACS, data were analyzed for statistical significance either by unpaired, two-tailed t-test, or one-way ANOVA with Bonferroni multiple comparison correction, unless indicated otherwise, as implemented in GraphPad Prism 5; *: $\mathrm{p}<0.05, * *: \mathrm{p}<0.001$, and $* * *: \mathrm{p}<0.0001$. For Nanostring, data was normalized using the geometric means of the NanoString positive controls and background levels were established using the means of the negative controls. Variance was similar between all compared groups. Housekeeping genes Tubb5, Hprt1, Bact, and Cltc were used to normalize between samples. Data was then log-transformed.

\section{Supplementary Material}

Refer to Web version on PubMed Central for supplementary material. 


\section{Acknowledgments}

This work was supported by the Juvenile Diabetes Research Foundation (JDRF) (Grant 17-2007-1063), and Leona M. and Harry B. Helmsley Charitable Trust Foundation (Grants 09PG-T1D027 and 2015PG-T1D063), National Institutes of Health (Grants EB000244, EB000351, DE013023 and CA151884), and through a generous gift from the Tayebati Family Foundation. JCD was supported by JDRF postdoctoral fellowship (Grant 3-PDF-2015-91-AN). OV was supported by JDRF and DOD/CDMRP postdoctoral fellowships (Grants 3-2013-178 and W81XWH-13-1-0215, respectively). J.O. is supported by the National Institutes of Health (NIH/NIDDK) R01DK091526 and the Chicago Diabetes Project. D.G. is supported by the National Institutes of Health (NIH/ NIDDK) UC4 DK104218. The authors would like to acknowledge the use of resources at W.M. Keck Biological Imaging Facility (Whitehead Institute), and Microscopy, Histology, Whole Animal Imaging, and Flow Cytometry Core Facilities (Swanson Biotechnology Center, David H. Koch Institute for Integrative Cancer Research at MIT).

\section{References}

1. Kurtz S, Ong K, Lau E, Mowat F, Halpern M. Projections of primary and revision hip and knee arthroplasty in the United States from 2005 to 2030. The Journal of bone and joint surgery American volume. 2007; 89(4):780-785. [PubMed: 17403800]

2. Med I. Medical Devices and the Public's Health: The FDA 510(k) Clearance Process at 35 Years. Medical Devices and the Public's Health: The Fda 510(K) Clearance Process at 35 Years. 2011:1298.

3. Cobelli N, Scharf B, Crisi GM, Hardin J, Santambrogio L. Mediators of the inflammatory response to joint replacement devices. Nature reviews Rheumatology. 2011; 7(10):600-608. [PubMed: 21894210]

4. Hubbell JA, Langer R. Translating materials design to the clinic. Nature materials. 2013; 12(11): 963-966. [PubMed: 24150414]

5. Fattahi P, Yang G, Kim G, Abidian MR. A review of organic and inorganic biomaterials for neural interfaces. Advanced materials. 2014; 26(12):1846-1885. [PubMed: 24677434]

6. Farra R, Sheppard NF Jr, McCabe L, Neer RM, Anderson JM, Santini JT Jr, et al. First-in-human testing of a wirelessly controlled drug delivery microchip. Sci Transl Med. 2012; 4(122):122ra121.

7. Rosen MR, Robinson RB, Brink PR, Cohen IS. The road to biological pacing. Nat Rev Cardiol. 2011; 8(11):656-666. [PubMed: 21844918]

8. Nichols SP, Koh A, Storm WL, Shin JH, Schoenfisch MH. Biocompatible materials for continuous glucose monitoring devices. Chemical reviews. 2013; 113(4):2528-2549. [PubMed: 23387395]

9. Perez-Cambrodi RJ, Pinero DP, Ferrer-Blasco T, Cervino A, Brautaset R. The posterior chamber phakic refractive lens (PRL): a review. Eye. 2013; 27(1):14-21. [PubMed: 23222559]

10. Kearney CJ, Mooney DJ. Macroscale delivery systems for molecular and cellular payloads. Nature materials. 2013; 12(11):1004-1017. [PubMed: 24150418]

11. Anderson JM, Rodriguez A, Chang DT. Foreign body reaction to biomaterials. Semin Immunol. 2008; 20(2):86-100. [PubMed: 18162407]

12. Wynn TA, Ramalingam TR. Mechanisms of fibrosis: therapeutic translation for fibrotic disease. Nature medicine. 2012; 18(7):1028-1040.

13. Kenneth Ward W. A Review of the Foreign-body Response to Subcutaneously-implanted Devices: The Role of Macrophages and Cytokines in Biofouling and Fibrosis. J Diabetes Sci Technol Online. 2008; 2:768-777.

14. Gordon S. Alternative activation of macrophages. Nat Rev Immunol. 2003; 3(1):23-35. [PubMed: 12511873]

15. Grainger DW. All charged up about implanted biomaterials. Nat Biotechnol. 2013; 31(6):507-509. [PubMed: 23752436]

16. Sussman EM, Halpin MC, Muster J, Moon RT, Ratner BD. Porous implants modulate healing and induce shifts in local macrophage polarization in the foreign body reaction. Annals of biomedical engineering. 2014; 42(7):1508-1516. [PubMed: 24248559]

17. Kyriakides TR, Foster MJ, Keeney GE, Tsai A, Giachelli CM, Clark-Lewis I, et al. The CC chemokine ligand, CCL2/MCP1, participates in macrophage fusion and foreign body giant cell formation. Am J Pathol. 2004; 165(6):2157-2166. [PubMed: 15579457] 
18. Rodriguez A, Meyerson H, Anderson JM. Quantitative in vivo cytokine analysis at synthetic biomaterial implant sites. Journal of biomedical materials research Part A. 2009; 89(1):152-159. [PubMed: 18431759]

19. Lee KY, Mooney DJ. Alginate: properties and biomedical applications. Progress in polymer science. 2012; 37(1):106-126. [PubMed: 22125349]

20. de Vos P, Faas MM, Strand B, Calafiore R. Alginate-based microcapsules for immunoisolation of pancreatic islets. Biomaterials. 2006; 27(32):5603-5617. [PubMed: 16879864]

21. Jacobs-Tulleneers-Thevissen D, Chintinne M, Ling Z, Gillard P, Schoonjans L, Delvaux G, et al. Sustained function of alginate-encapsulated human islet cell implants in the peritoneal cavity of mice leading to a pilot study in a type 1 diabetic patient. Diabetologia. 2013; 56(7):1605-1614. [PubMed: 23620058]

22. Tuch BE, Keogh GW, Williams LJ, Wu W, Foster JL, Vaithilingam V, et al. Safety and viability of microencapsulated human islets transplanted into diabetic humans. Diabetes care. 2009; 32(10): 1887-1889. [PubMed: 19549731]

23. Weir GC. Islet encapsulation: advances and obstacles. Diabetologia. 2013; 56(7):1458-1461. [PubMed: 23636639]

24. Dang TT, Thai AV, Cohen J, Slosberg JE, Siniakowicz K, Doloff JC, et al. Enhanced function of immuno-isolated islets in diabetes therapy by co-encapsulation with an anti-inflammatory drug. Biomaterials. 2013; 34(23):5792-5801. [PubMed: 23660251]

25. Robitaille R, Dusseault J, Henley N, Desbiens K, Labrecque N, Halle JP. Inflammatory response to peritoneal implantation of alginate-poly-L-lysine microcapsules. Biomaterials. 2005; 26(19):41194127. [PubMed: 15664639]

26. Vegas AJ, Veiseh O, Doloff JC, Ma M, Tam HH, Bratlie K, et al. Combinatorial hydrogel library enables identification of materials that mitigate the foreign body response in primates. Nat Biotechnol. 2016; 34(3):345-352. [PubMed: 26807527]

27. Veiseh O, Doloff JC, Ma M, Vegas AJ, Tam HH, Bader AR, et al. Size- and shape-dependent foreign body immune response to materials implanted in rodents and non-human primates. Nature materials. 2015; 14(6):643-651. [PubMed: 25985456]

28. Harding JL, Reynolds MM. Combating medical device fouling. Trends in biotechnology. 2014; 32(3):140-146. [PubMed: 24438709]

29. Langer R. Perspectives and challenges in tissue engineering and regenerative medicine. Advanced materials. 2009; 21(32-33):3235-3236. [PubMed: 20882493]

30. Rhen T, Cidlowski JA. Antiinflammatory action of glucocorticoids--new mechanisms for old drugs. New England Journal of Medicine. 2005; 353(16):1711. [PubMed: 16236742]

31. Khan, W., Muntimadugu, E., Jaffe, M., Domb, AJ. Focal Controlled Drug Delivery. Springer; US: 2014. Implantable Medical Devices; p. 33-59.

32. Attur MG, Patel R, Thakker G, Vyas P, Levartovsky D, Patel P, et al. Differential antiinflammatory effects of immunosuppressive drugs: cyclosporin, rapamycin and FK-506 on inducible nitric oxide synthase, nitric oxide, cyclooxygenase-2 and PGE 2 production. Inflammation Research. 2000; 49(1):20-26. [PubMed: 10778917]

33. King A, Sandler S, Andersson A. The effect of host factors and capsule composition on the cellular overgrowth on implanted alginate capsules. Journal of biomedical materials research. 2001; 57(3): 374-383. [PubMed: 11523032]

34. Manoury B, Caulet-Maugendre S, Guenon I, Lagente V, Boichot E. TIMP-1 is a key factor of fibrogenic response to bleomycin in mouse lung. International journal of immunopathology and pharmacology. 2006; 19(3):471-487. [PubMed: 17026855]

35. Paredes-Juarez GA, de Haan BJ, Faas MM, de Vos P. The role of pathogen-associated molecular patterns in inflammatory responses against alginate based microcapsules. J Control Release. 2013; 172(3):983-992. [PubMed: 24051034]

36. Jhunjhunwala S, Aresta-DaSilva S, Tang K, Alvarez D, Webber MJ, Tang BC, et al. Neutrophil Responses to Sterile Implant Materials. PloS one. 2015; 10(9):e0137550. [PubMed: 26355958]

37. Shi C, Pamer EG. Monocyte recruitment during infection and inflammation. Nat Rev Immunol. 2011; 11(11):762-774. [PubMed: 21984070] 
38. Wood KJ, Bushell A, Hester J. Regulatory immune cells in transplantation. Nat Rev Immunol. 2012; 12(6):417-430. [PubMed: 22627860]

39. Bratlie KM, Dang TT, Lyle S, Nahrendorf M, Weissleder R, Langer R, et al. Rapid biocompatibility analysis of materials via in vivo fluorescence imaging of mouse models. PloS one. 2010; 5(4):e10032. [PubMed: 20386609]

40. Ito M, Hiramatsu H, Kobayashi K, Suzue K, Kawahata M, Hioki K, et al. NOD/SCID/gamma(c) (null) mouse: an excellent recipient mouse model for engraftment of human cells. Blood. 2002; 100(9):3175-3182. [PubMed: 12384415]

41. Diel IJ, Bergner R, Grotz KA. Adverse effects of bisphosphonates: current issues. The journal of supportive oncology. 2007; 5(10):475-482. [PubMed: 18240669]

42. Pyonteck SM, Akkari L, Schuhmacher AJ, Bowman RL, Sevenich L, Quail DF, et al. CSF-1R inhibition alters macrophage polarization and blocks glioma progression. Nat Med. 2013; 19(10): 1264-1272. [PubMed: 24056773]

43. Conway JG, McDonald B, Parham J, Keith B, Rusnak DW, Shaw E, et al. Inhibition of colonystimulating-factor-1 signaling in vivo with the orally bioavailable cFMS kinase inhibitor GW2580. Proceedings of the National Academy of Sciences of the United States of America. 2005; 102(44): 16078-16083. [PubMed: 16249345]

44. Laskin DL, Sunil VR, Gardner CR, Laskin JD. Macrophages and tissue injury: agents of defense or destruction? Annual review of pharmacology and toxicology. 2011; 51:267-288.

45. Arnold L, Perrin H, de Chanville CB, Saclier M, Hermand P, Poupel L, et al. CX3CR1 deficiency promotes muscle repair and regeneration by enhancing macrophage ApoE production. Nature communications. 2015; 6:8972.

46. Pesce J, Kaviratne M, Ramalingam TR, Thompson RW, Urban JF Jr, Cheever AW, et al. The IL-21 receptor augments Th2 effector function and alternative macrophage activation. The Journal of clinical investigation. 2006; 116(7):2044-2055. [PubMed: 16778988]

47. Carlsen HS, Baekkevold ES, Morton HC, Haraldsen G, Brandtzaeg P. Monocyte-like and mature macrophages produce CXCL13 (B cell-attracting chemokine 1) in inflammatory lesions with lymphoid neogenesis. Blood. 2004; 104(10):3021-3027. [PubMed: 15284119]

48. Affara NI, Ruffell B, Medler TR, Gunderson AJ, Johansson M, Bornstein S, et al. B cells regulate macrophage phenotype and response to chemotherapy in squamous carcinomas. Cancer cell. 2014; 25(6):809-821. [PubMed: 24909985]

49. Vuga LJ, Tedrow JR, Pandit KV, Tan J, Kass DJ, Xue J, et al. C-X-C motif chemokine 13 (CXCL13) is a prognostic biomarker of idiopathic pulmonary fibrosis. American journal of respiratory and critical care medicine. 2014; 189(8):966-974. [PubMed: 24628285]

50. Neale SD, Athanasou NA. Cytokine receptor profile of arthroplasty macrophages, foreign body giant cells and mature osteoclasts. Acta orthopaedica Scandinavica. 1999; 70(5):452-458. [PubMed: 10622477] 
a

Phase Contrast

b

DAPI

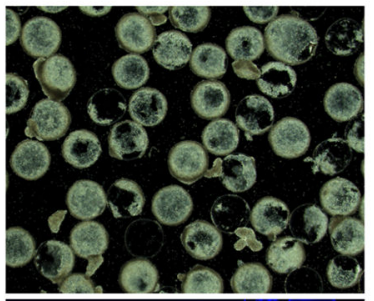

d

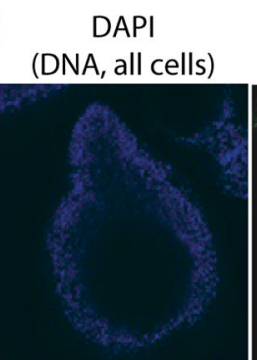

DAPI

e

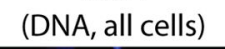

C

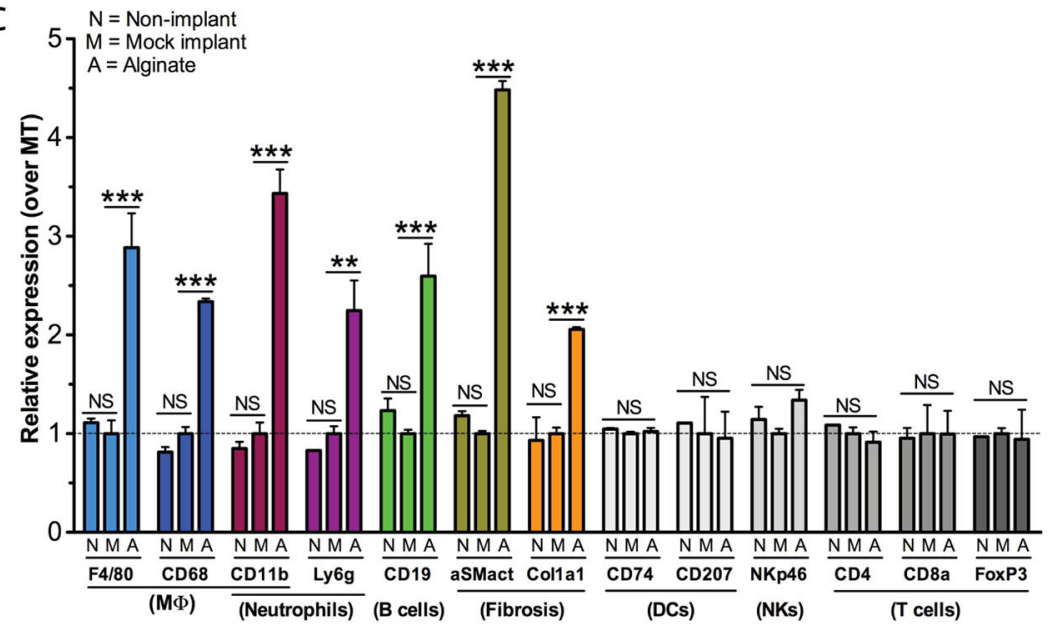

CD68

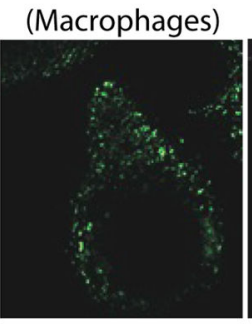

Ly6g (Gr1) (Neutrophils)

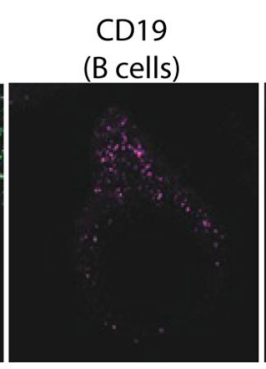

aSMactin (Fibrosis)

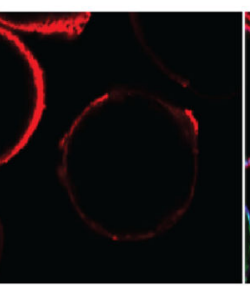

aSMactin (Fibrosis)

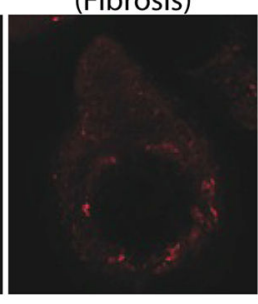

Overlay

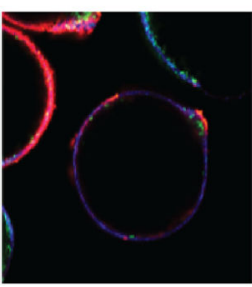

Overlay

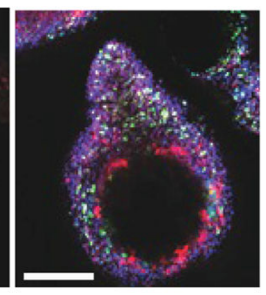

Brightfield

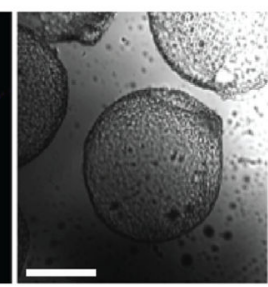

f

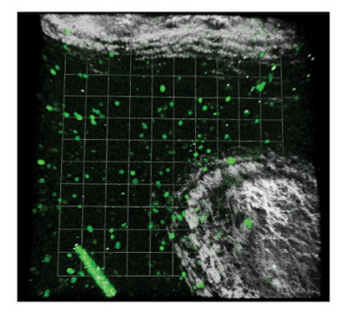

g

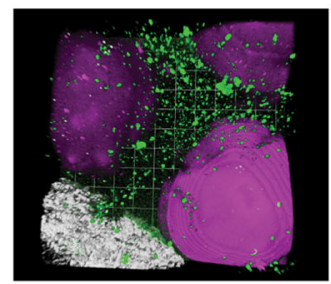

Figure 1. Numerous immune populations respond to implanted alginate spheres

SLG20 alginate $500 \mu \mathrm{m}$ diameter spheres $(0.35 \mathrm{ml}$ total implant volume $)$ were implanted into the intraperitoneal space of C57BL/6 mice, where they were retained for 14 days and analyzed for degree of fibrosis upon retrieval. (a) Dark field phase contrast image and (b) DAPI immunofluorescence image obtained from retrieved spheres reveal a significant level of cellular overgrowth; scale bar $=2000 \mu \mathrm{m}$ ). (c) qPCR analysis of innate and adaptive immune population and fibrosis markers present on fibrosed alginate spheres, A, or nonimplant, $\mathrm{N}$, or mock implant, $\mathrm{M}$, omental and epididymal fat pad tissue after 14 days postimplantation into the IP space of C57BL/6 mice. $\mathrm{M} \phi=$ macrophages, DCs $=$ Dendritic cells, $\mathrm{NKs}=$ Natural Killer cells. Data: mean \pm SEM, $\mathrm{n}=5$. qPCR statistical analysis: one-way ANOVA with Bonferroni multiple comparison correction **: $\mathrm{p}<0.001$, and $* * *$ : $\mathrm{p}$ 0.0001; ns = not significantly different. (d) Confocal staining showing DAPI (cellular nuclei), innate immune macrophage marker CD68 (green), adaptive immune B cells (magenta), alpha smooth muscle actin (aSMactin, myofibroblasts, red), and overlay making up the fibrosis on $500 \mu \mathrm{m}$ alginate spheres. Scale bar $=400 \mu \mathrm{m}$. (e) Confocal staining showing DAPI (cellular nuclei), innate immune neutrophil marker Ly6g/Gr1 (green), alpha 
smooth muscle actin (aSMactin, myofibroblasts, red), fluorescent overlay, and brightfield image for the fibrosis on $500 \mu \mathrm{m}$ alginate spheres. Scale bar $=400 \mu \mathrm{m}$. In vivo intravital imaging of adaptive B cell behavior and accumulation at day 14 post-implant for mock implant (f) or SLG20 sphere (pink) implanted (g) C57BL/6-Ccr6 (EGFP) mice. For intravital imaging: $n=3$ mice per treatment. For all others, $n=5$ mice per group. Experiments repeated at least 3 times. 
a)

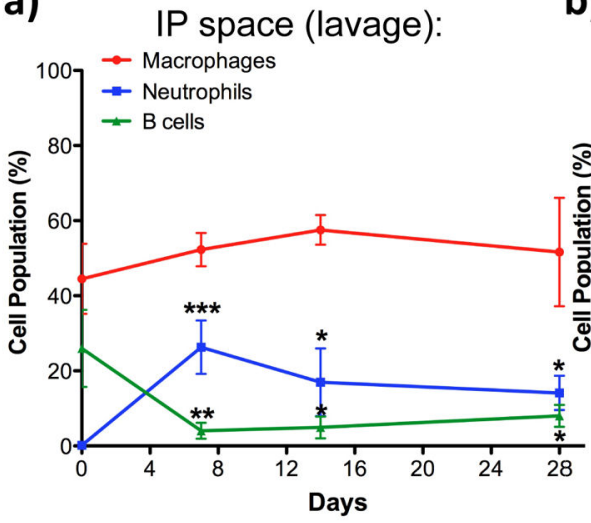

b)

Fibrosed tissue (infiltration):

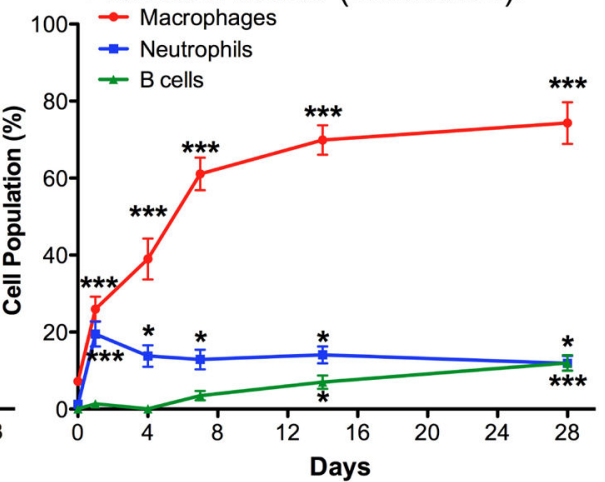

c)

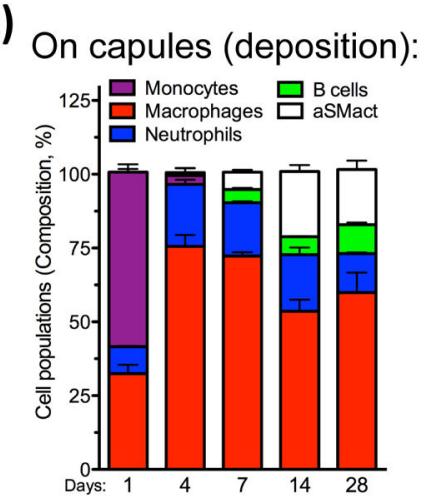

d)

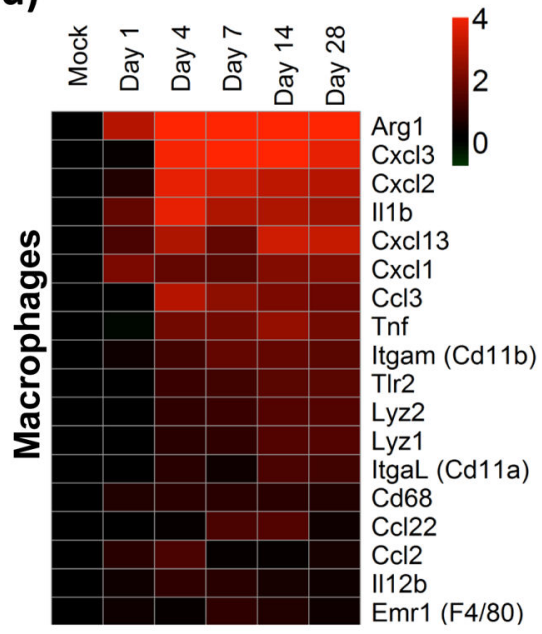

e)

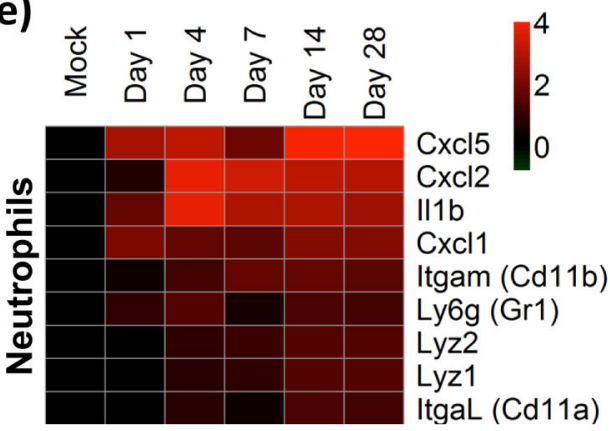

f)

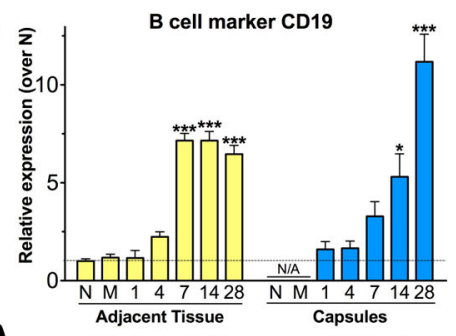

g)

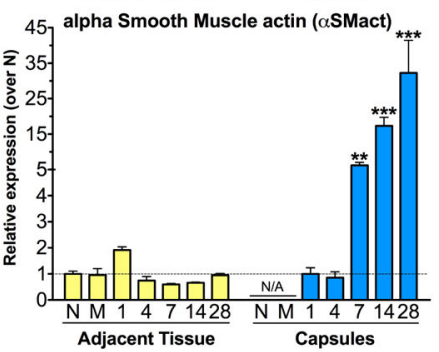

Figure 2. The immune response to implanted biomaterial alginate is long lived

Flow analysis, using specific markers for responding host innate immune macrophage (red), neutrophil (blue), and adaptive immune B cells (green) at 1, 4, 7, 14, and 28 days postimplantation among peritoneal exudate (lavage) (a), adjacent fibrosed omental and epididymal fat pads (through which host cells infiltrate and to which material spheres become fibrosed, becoming part of the fibrosis themselves) (b), and directly on fibrosed alginate spheres (c). For sphere-specific FACS, fixation and permeabilization was also carried out to stain for fibrosis-depositing myofibroblasts (aSMactin, white). See supplemental methods section for all antibody combinations. NanoString-based analysis enrichment for expression of significantly increasing macrophage (d) and neutrophil (e) associated physical and cytokine markers analyzed from deposited cell RNA extracts at 14 days post-implant: fold changes between samples, presented on a base 2 logarithmic scale. (f) qPCR analysis on either adjacent fibrosed tissue (yellow) or fibrotic spheres alone (blue), relative to day 1 tissue for all, showing that there is a slightly delayed mobilization of adaptive B cells out of adjacent fibrosed tissue and onto spheres after 1 week postimplantation. (g) qPCR analysis on either adjacent fibrosed tissue (yellow) or spheres alone (blue), relative to day 1 for either tissue or spheres, respectively, showing that there is no apparent response (due to high tissue background) across time points for aSMactin, as 
opposed to a high dynamic response if spheres alone are examined. Error bars, mean \pm SEM. For all analyses $n=5$ per treatment. FACS and qPCR experiments were performed twice and NanoString analysis was performed once. FACS time point comparisons were performed by unpaired, two-tailed t-test *: $\mathrm{p}<0.05$, **: $\mathrm{p}<0.001$, and ***: $\mathrm{p}<0.0001$, vs mock (M) or non-implanted (N) controls. For NanoString statistical analyses see supplemental methods. 
a)

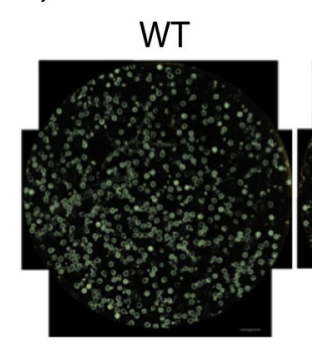

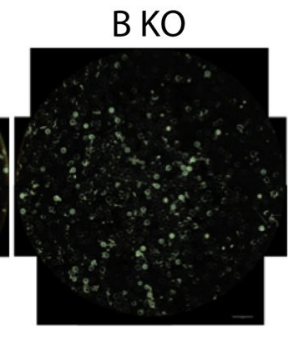

b)

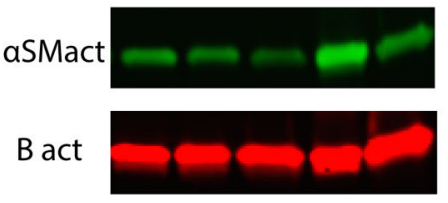

c)

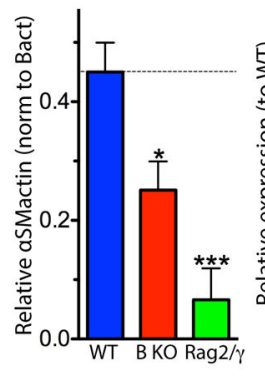

d)

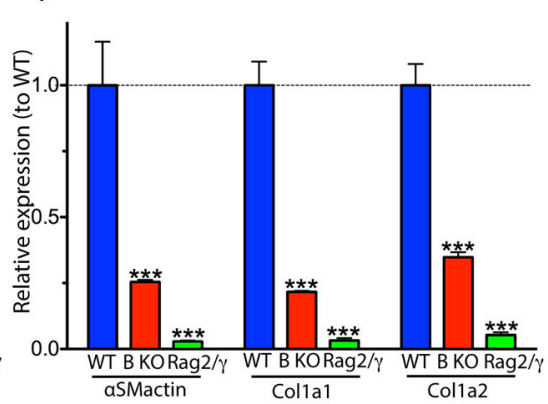

$\mathrm{T} \& \mathrm{~B} K \mathrm{KO}$

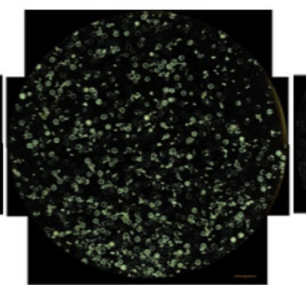

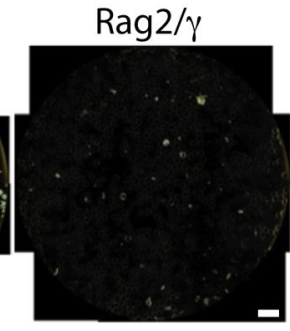

f)

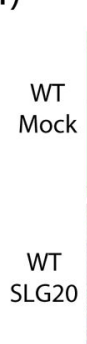

H\&E

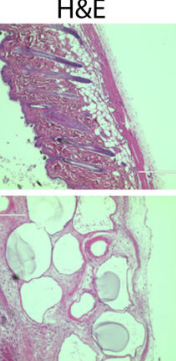

Masson's Trichrome

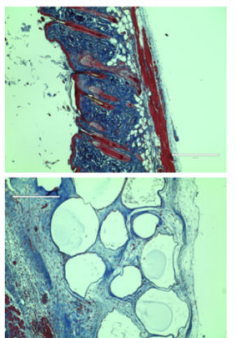

B KO

$\operatorname{Rag} 2 / \gamma$
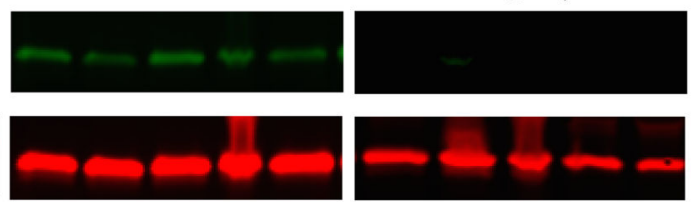

SLG20

e)

g)

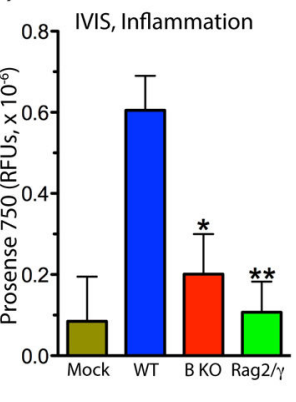

\section{)}

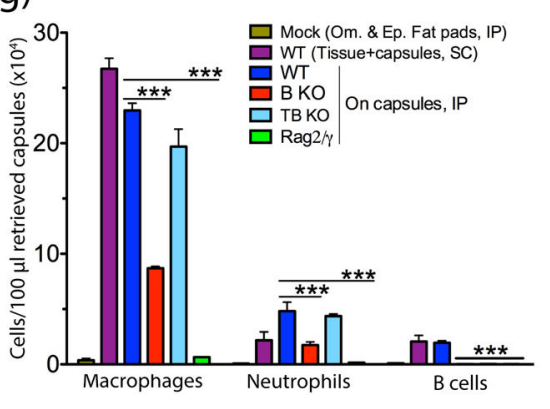

Figure 3. Innate immune macrophage function is required for fibrosis of alginate SLG20 alginate $500 \mu \mathrm{m}$ spheres $(0.35 \mathrm{ml})$ were implanted for 14 days into the intraperitoneal (IP) or subcutaneous (SC) space of wild type and various knockout (IghM ${ }^{\text {mull, }}$, B cell deficient; Rag $2^{\text {null }}, \mathrm{T}$ and B cell deficient; and Rag $2^{\text {null }} / I L 2 r \gamma^{\text {null }}, \mathrm{T}, \mathrm{B}, \mathrm{NK}$ cell deficient, and $\mathrm{M} \phi$ and DC dysfunctional) C57BL/6 mice, and analyzed for degree of fibrosis upon retrieval. (a) Phase contrast images showing that fibrosis, as compared to wild type (WT) response, was partially decreased upon removal of adaptive B cells (B KO), restored when $\mathrm{T}$ cells were also removed (TB KO), and completely removed with additional $\mathrm{M} \phi$ dysfunction (Rag2/ $\gamma$ KO). Scale bar $=2000 \mu \mathrm{m}$. (b) Western blot analysis for fibrosisassociated myofibroblast $\mathbf{a}$-smooth muscle actin ( $\mathbf{a}$-SMact) expression in microsphere overgrowth (5 individual mice/group), confirming relative decreases in fibrosis, as observed in (a). (c) Plot of western blot band intensities from (b). (d) Relative q-PCR expression analysis for markers of fibrotic capsule formation on material implants: a-SMactin, Collagen 1a1 (Col1a1), and Collagen 1a2 (Col1a2), on retrieved spheres from various C57BL/6 strains (as shown). (e) Quantified IVIS imaging fluorescent ProSense 750 inflammation levels across Mock (saline), WT, B KO, and Rag2/ $\gamma$ KO mice 7 days post-SC implant. (f) H\&E and Masson's Trichrome stained histological sections of excised SC tissue 14 days post-implant from various strains (Scale bar $=500 \mu \mathrm{m}$ ). $(\mathrm{g})$ Flow analysis for responding macrophages, neutrophils, and B cells dissociated from spheres (100 $\mu \mathrm{l}$ material in all cases) 14 days post-IP implantation (note: a WT SC group is included for comparison). 
qPCR, western blot, IVIS, and FACS statistical analysis: one-way ANOVA with Bonferroni multiple comparison correction *: $\mathrm{p}<0.05$, **: $\mathrm{p}<0.001$, and $* * * \mathrm{p}<0.0001$, vs WT.

Error bars, mean \pm SEM. $n=5$ mice/group for all assays. Experiments repeated at least $2-3$ times. 
a)

b)

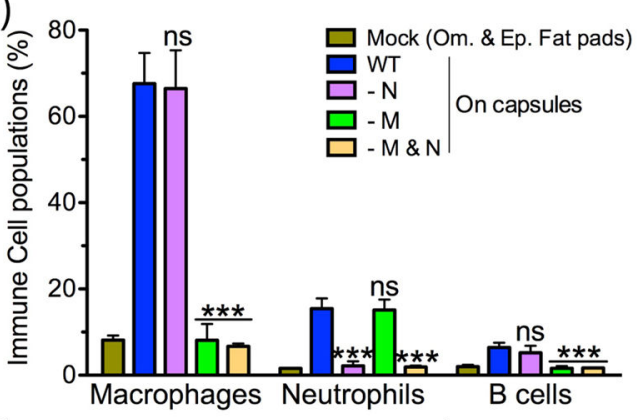

d)

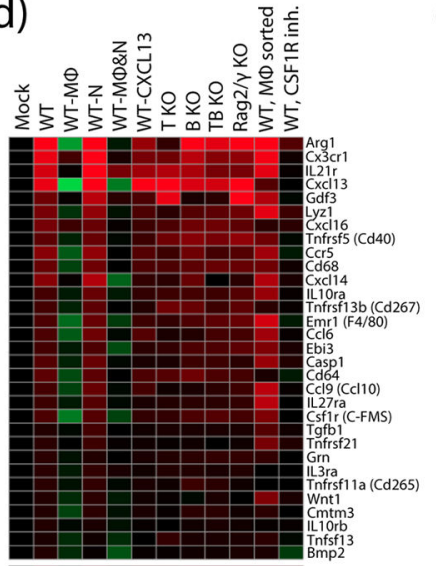

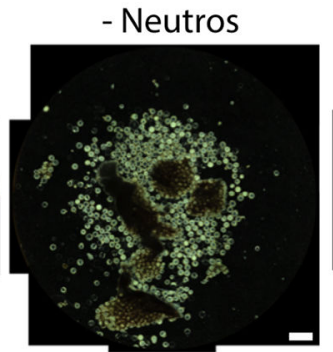

Mock (Om. \& Ep. Fat pads) $M \& N$

\section{e)}

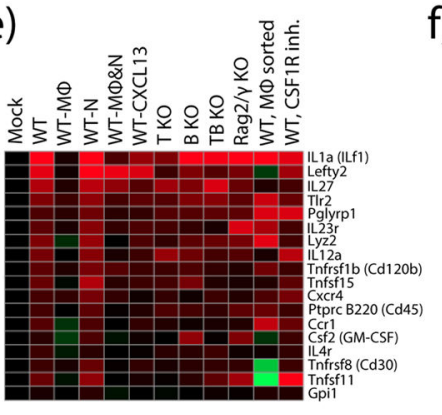

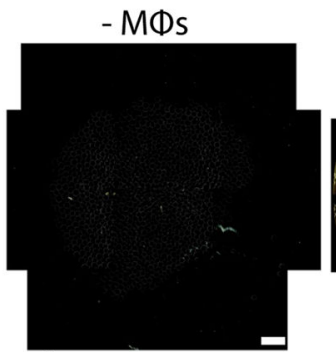

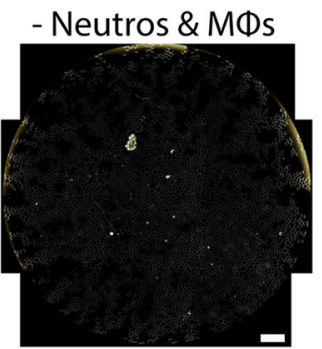

C)

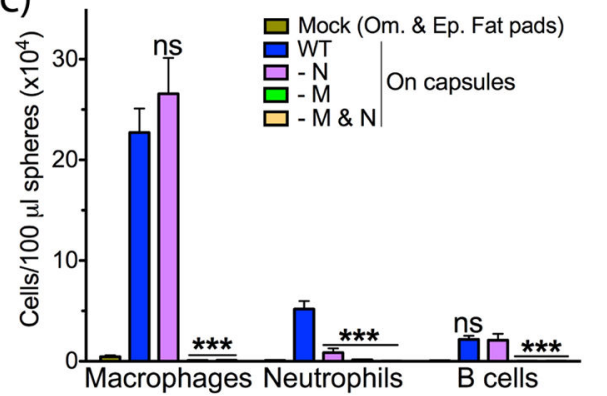

f)

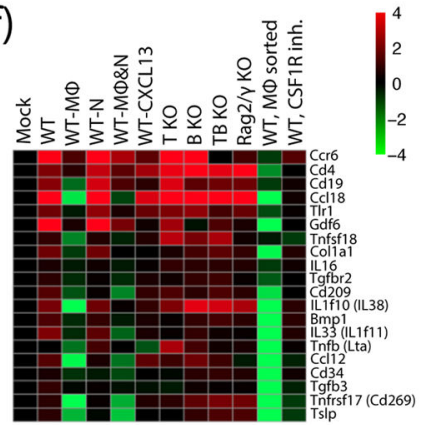

Figure 4. Macrophages, and not neutrophils, are required for fibrosis of alginate

SLG20 alginate $500 \mu \mathrm{m}$ diameter spheres $(0.35 \mathrm{ml})$ were implanted into the intraperitoneal (IP) space of wild type C57BL/6 mice, either treated with vehicle (WT) or targeted depletion agents to eliminate macrophages and/or neutrophils over a 14-day implantation (see supplemental methods for dosing), and analyzed for degree of fibrosis upon retrieval. (a) Phase contrast images showing that fibrosis, as compared to WT, was completely eliminated following removal of innate immune macrophages (- M $\phi s)$, either alone or in combination with neutrophil depletion (- Neutros \& M $\phi s$ ). Neutrophil depletion alone (- Neutros) did not alleviate fibrosis, instead augmenting sphere clumping. Scale bar $=2000 \mu \mathrm{m}$. (b) Flow analysis for responding host immune macrophages, neutrophils, and B cells dissociated directly from spheres (as percent composition) 14 days post-IP implantation, illustrating specificity of depletions. Recruited downstream of macrophages, adaptive B cells also decreased following macrophage depletion. (c) Flow analysis in (b) normalized to cell counts from $100 \mu \mathrm{l}$ material (in each case) showing absolute cell presence, and the lack thereof on spheres in macrophage-depleted mice. NanoString expression analysis of all known cytokine and cytokine receptors (see Supplemental Figure S12 for complete data set, 
excerpted based on response here) for macrophage-specific factors (determined by depletion and cell sorting) also inhibited by GW2580 (d), for macrophage-specific factors not affected by CSF1R inhibition, suggesting altered macrophage polarization/phenotype and residual function (e), and for macrophage-associated factors (by depletion, but NOT cell sorting) also inhibited by the CSF1R inhibitor GW2580 (f), analyzed from RNA extracts at 14 days postimplant, presented on a base 2 logarithmic scale. Since these factors were removed by macrophage depletion, but not expressed by sorted macrophages (decreased/diluted green expression profile as compared to mock, whole-tissue controls), they likely belong to cells that are recruited by macrophages in the downstream fibrotic cascade (ie, CD19, B cell marker and fibrosis protein collagen 1a1, Col1a1). FACS statistical analysis: one-way ANOVA with Bonferroni multiple comparison correction ***: $\mathrm{p}<0.0001$, vs WT. ns $=$ not significantly different. $\mathrm{n}=5$ mice/group. Experiments repeated at least 3 times. 
a)
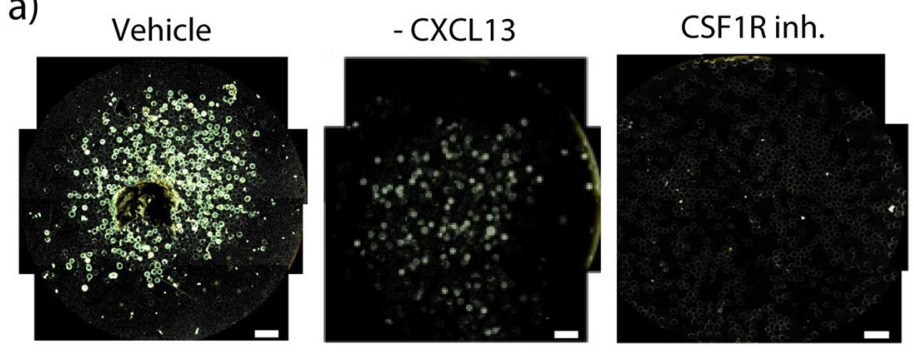

b)

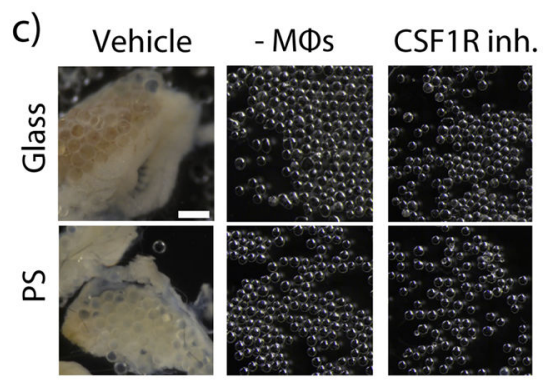

d)
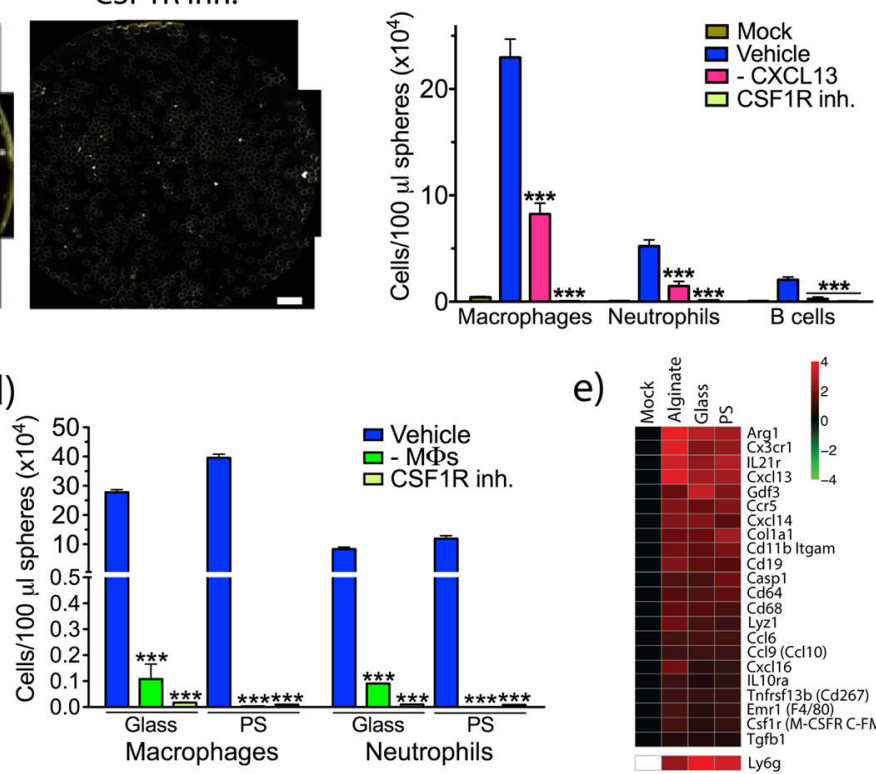

e)
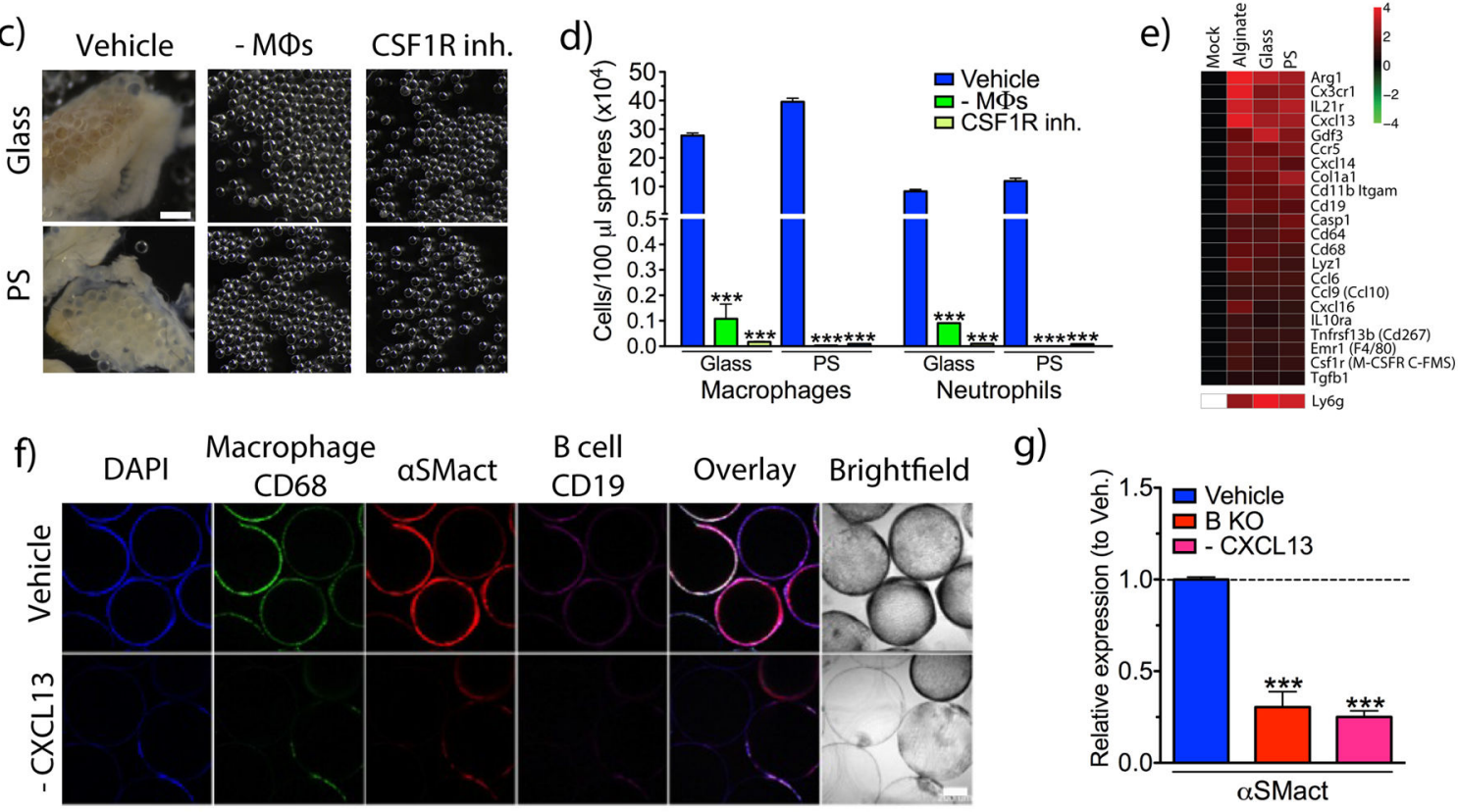

g)

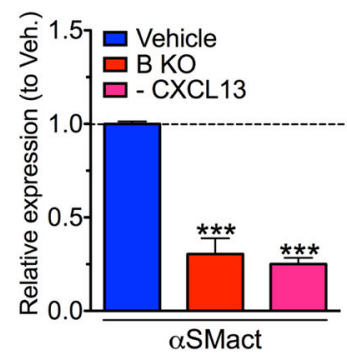

Figure 5. CSF1R-dependent macrophages recruit fibrosis-potentiating B cells via CXCL13 CSF1R inhibition prevents the entire (innate and adaptive) immune response to implanted biomaterials. Phase contrast images showing that fibrosis, as compared to wild type (WT) vehicle-treated control, was partially eliminated by CXCL13 neutralization, and completely eliminated with continuous CSF1R inhibition (inh.; 160 mg/kg BW GW2580 SC, daily) over a 14-day implant period (a). Scale bar $=2000 \mu \mathrm{m}$. Fibrosis was reduced the same extent as that of the B cell knockout (B KO) shown in Figure 3a. (b) Flow analysis for responding macrophages, neutrophils, and B cells dissociated directly from spheres (100 $\mu$ material in all cases) 14 days post-intraperitoneal (IP) implant, showing partial loss of cell presence with CXCL13 neutralization, and complete loss following CSF1R inhibition. (c) Brightfield images showing that fibrosis, compared to vehicle control, was completely eliminated by both macrophage depletion (- M $\phi$ s) and CSF1R inhibition. Scale bar $=1000 \mu \mathrm{m}$. (d) Flow analysis for responding host innate immune macrophages and neutrophils dissociated directly from spheres (100 $\mu$ l of each material) 14 days post-IP implantation, showing the loss of immune adhesion with loss of fibrosis due to either macrophage depletion (- M $\phi s)$ or CSF1R inhibition. (e) NanoString expression analysis for all known cytokine and cytokine receptors (see Supplemental Figure S18 for complete data set, excerpted here), showing similar unique factors increased across all material (hydrogel alginate, ceramic glass, and 
polymer polystyrene (PS)) groups. (f) Confocal for DAPI (cellular nuclei), macrophage marker CD68 (green), B cell marker CD19 (magenta), fibrosis-associated myofibroblast marker alpha smooth muscle actin (aSMactin, myofibroblasts, red), overlay, and brightfield imaging, showing that CXCL13 neutralization resulted in loss of B cell recruitment. Scale bar $=200 \mu \mathrm{m}$. (g) qPCR expression analysis of a-SMactin directly on retrieved spheres from Vehicle-treated (WT), B KO, and CXCL13-neutralized WT mice, plotted relative to expression levels on spheres from WT mice. Statistical analysis: one-way ANOVA with Bonferroni multiple comparison correction $* * *$ : $\mathrm{p}<0.0001$, vs Vehicle. $\mathrm{n}=5$ mice/group. Experiments repeated at least 2-3 times. 
a)
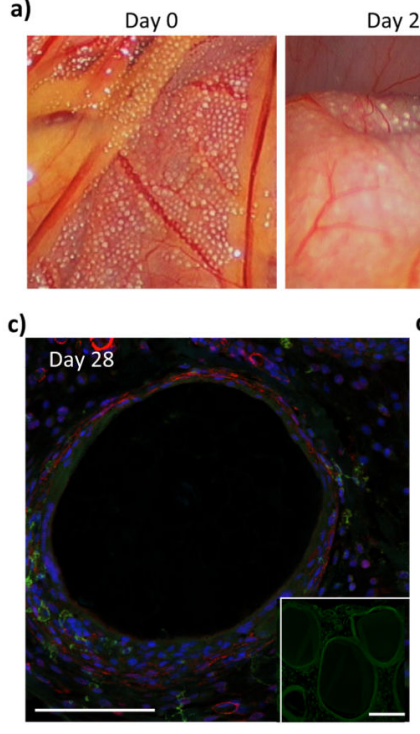

Day 28

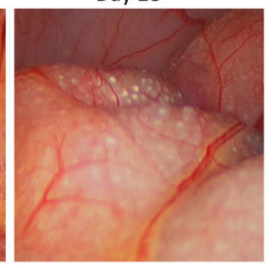

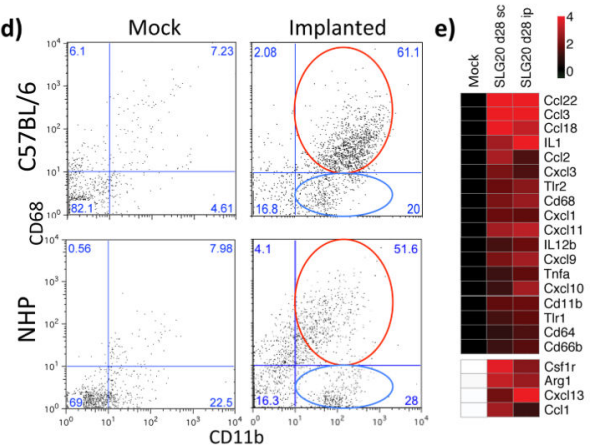

b)

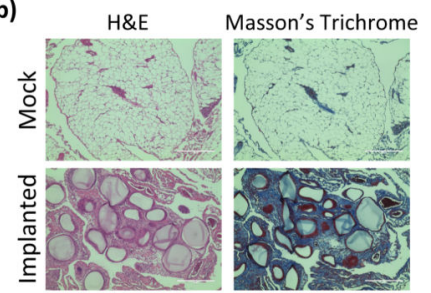

Figure 6. Essential fibrotic cascade players are also increased in non-human primates $0.5 \mathrm{~mm}$-sized SLG20 hydrogel spheres of were implanted into either the intraperitoneal (IP) or subcutaneous (SC) dorsal region of cynomolgus monkeys and retrieved by laparoscopyguided tissue excision or biopsy punch after 28 days $^{27}$. (a) Laparoscopy images taken on days 0 and 28 (initial implantation and retrieval from the IP space). (b) H\&E and Masson's Trichrome stained histological sections of excised IP omentum tissue at 28 days for nonfibrosed fat-laden (No material, Mock) or heavily collagen-deposited and sphere-embedded (Implanted) omental tissue. Scale bars $=400 \mu \mathrm{m}$. (c) Main figure: Confocal staining showing DAPI (cellular nuclei), innate immune macrophage marker CD68 (green), and fibrosis-associated activated myofibroblast alpha smooth muscle actin (aSMactin) staining (red), showing cellular infiltration around and fibrosis deposition on an embedded $500 \mu \mathrm{m}$ alginate sphere; Inset: additional confocal for CSF1R (green), showing positive staining on not only more distant macrophages but also material-proximal and fused foreign body giant cells (FBGCs); both 20x magnification. White scale bars: both $200 \mu \mathrm{m}$ for each respective image. (d) Flow analysis showing similar host innate immune macrophage $\left(\mathrm{CD} 68^{+} \mathrm{CD} 11 \mathrm{~b}^{+}\right.$, top right quadrants) and remaining neutrophil/myeloid $\left(\mathrm{CD}^{-} 8^{-} \mathrm{CD} 11 \mathrm{~b}^{+}\right.$, bottom right quadrants) cells across C57BL/6 mice and cynomolgus monkeys, dissociated directly from fibrosed spheres and adjacent fibrosed omentum tissue (as percent composition) 28 days post-IP implantation. While the prominence of CD11b seems to be inverted in C57BL/6 mice vs cynomolgus monkeys, population response percentages are similar 28 days post-IP implantation. (e) NanoString analysis for immune markers and cytokines, originally identified in C57BL/6 mice (Note: CD66b is used here as a neutrophil marker, as Ly6g/Gr1 does not exist in NHPs or humans). Significant increases are observed for macrophage markers, as well as CSF1R and CXCL13 in both peritoneal and subcutaneous implant sites, as compared to mock (saline-injected) controls (there was no difference between SC and IP mock controls). $\mathrm{N}=2$ for IP-implanted groups; $\mathrm{N}=4$ for subcutaneous (SC) treatment groups. These experiments were performed once for SC and twice for IP delivery. 